\title{
Atomic data for neutron-capture elements
}

\section{Charge transfer rate coefficients for low-charge ions of $\mathrm{Ge}, \mathrm{Se}, \mathrm{Br}, \mathrm{Kr}, \mathrm{Rb}$, and $\mathrm{Xe}^{\star}$}

\author{
N. C. Sterling ${ }^{1, \star \star}$ and P. C. Stancil ${ }^{2}$ \\ ${ }^{1}$ Michigan State University, Department of Physics and Astronomy, 3248 Biomedical Physical Sciences, East Lansing, MI, \\ 48824-2320, USA \\ e-mail: sterling@pa.msu.edu \\ 2 Department of Physics and Astronomy and the Center for Simulational Physics, University of Georgia, Athens, \\ GA 30602-2451, USA \\ e-mail: stancil@physast.uga.edu
}

Received 28 June 2011 / Accepted 12 August 2011

ABSTRACT

\begin{abstract}
We present total and final-state resolved charge transfer (CT) rate coefficients for low-charge $\mathrm{Ge}, \mathrm{Se}, \mathrm{Br}, \mathrm{Kr}, \mathrm{Rb}$, and Xe ions reacting with neutral hydrogen over the temperature range $10^{2}-10^{6} \mathrm{~K}$. Each of these elements has been detected in ionized astrophysical nebulae, particularly planetary nebulae. CT rate coefficients are a key ingredient for the ionization equilibrium solutions needed to determine total elemental abundances from those of the observed ions. A multi-channel Landau Zener approach was used to compute rate coefficients for projectile ions with charges $q=2-5$, and for singly-charged ions the Demkov approximation was utilized. Our results for five-times ionized species are lower limits, due to the incompleteness of level energies in the NIST database. In addition, we computed rate coefficients for charge transfer ionization reactions between the neutral species of the above six elements and ionized hydrogen. The resulting total and state-resolved CT rate coefficients are tabulated and available at the CDS. In tandem with our concurrent investigations of other important atomic processes in photoionized nebulae, this work will enable robust investigations of neutron-capture element abundances and nucleosynthesis via nebular spectroscopy.
\end{abstract}

Key words. atomic data - atomic processes - HII regions - nuclear reactions, nucleosynthesis, abundances - stars: evolution planetary nebulae: general

\section{Introduction}

The detection of $n$-capture elements (atomic number $Z>30$ ) in ionized astrophysical nebulae has underscored the need for atomic data required to accurately solve for the ionization equilibrium of these species. Trans-iron element emission lines have been detected in objects ranging from planetary nebulae (e.g., Zhang et al. 2005; Sharpee et al. 2007; Sterling et al. 2007; Sterling \& Dinerstein 2008; Sterling et al. 2009; Otsuka et al. 2010, 2011) to H II regions (e.g., Baldwin et al. 2000; Blum \& McGregor 2008, and references therein), and the interstellar medium of other galaxies (Vanzi et al. 2008). Determinations of their abundances have important implications for the production and chemical evolution of $n$-capture nuclides in the Universe (e.g., Busso et al. 1999; Sneden et al. 2008; Sterling \& Dinerstein 2008). Se, Kr, and Xe are the most widely detected $n$-capture elements in ionized nebulae (e.g, Dinerstein 2001; Sharpee et al. 2007; Sterling \& Dinerstein 2008; Sterling et al. 2009), but other trans-iron elements have also been detected in nebular spectra, including Ge (Sterling et al. 2002; Sterling \& Dinerstein 2003), $\mathrm{Br}$, and $\mathrm{Rb}$ (Péquignot \& Baluteau 1994; Zhang et al. 2005; Sharpee et al. 2007; Sterling et al. 2009).

\footnotetext{
* Tables 4 and 5 are only available in electronic form at the CDS via anonymous ftp to cdsarc.u-strasbg. fr (130.79.128.5) or via http://cdsarc.u-strasbg.fr/viz-bin/qcat?J/A+A/535/A117 $\star \star$ NSF Astronomy and Astrophysics Postdoctoral Fellow.
}

Due to the limited number of detectable $n$-capture element ions in individual nebulae, it is necessary to convert abundances of the observed ions to elemental abundances by correcting for unobserved ionization stages. Numerical simulations of ionized nebulae present a robust and accurate method of deriving such ionization corrections, provided that accurate data for atomic processes affecting the ionization equilibrium of the modeled elements are available. Since $n$-capture element emission lines have been detected primarily in photoionized nebulae, the most important atomic data required for ionization balance determinations are photoionization cross sections and rate coefficients for radiative recombination, dielectronic recombination, and charge transfer (CT). Unfortunately, these data are poorly if at all known for ions of trans-iron elements.

This paper is the third in a series presenting theoretical atomic data determinations relevant to nebular ionization balance solutions for low-charge $n$-capture element ions. In the first two (Sterling \& Witthoeft 2011; Sterling 2011), we furnished multi-configuration Breit-Pauli distorted-wave photoionization cross sections and radiative and dielectronic recombination rate coefficients for $\mathrm{Se}$ and $\mathrm{Kr}$ ions.

To address the need for $\mathrm{CT}$ data for low-charge ions of $n$-capture elements, we present calculations based on the Landau-Zener (LZ) and Demkov approximations. We consider reactions of the form

$\mathrm{X}^{q+}+\mathrm{H} \rightleftharpoons \mathrm{X}^{(q-1)+}+\mathrm{H}^{+}+\Delta \mathrm{E}$ 
with $q=1-5$ and $\mathrm{X}=\mathrm{Ge}, \mathrm{Se}, \mathrm{Br}, \mathrm{Kr}, \mathrm{Rb}$, and $\mathrm{Xe}$. The forward reaction, $\mathrm{CT}$ recombination (in terms of the projectile $\mathrm{X}^{q+}$ ), is exothermic for these elements when $q \geq 2$, and the reverse reaction for multiply-charged systems is generally unimportant at the temperatures of photoionized nebulae (near $10^{4} \mathrm{~K}$ ). On the other hand, of these elements only $\mathrm{Kr}$ has a higher ionization potential than $\mathrm{H}$, and hence $\mathrm{CT}$ recombination is endoergic for singly-ionized $\mathrm{Ge}, \mathrm{Se}, \mathrm{Br}, \mathrm{Rb}$, and $\mathrm{Xe}$. We therefore provide rate coefficients for both CT recombination of singly-charged ions and CT ionization of neutral species.

Our LZ calculations are multi-channel, in the sense that several exit channels for the $\mathrm{X}^{(q-1)+}$ system are simultaneously considered. This avoids the problem of overestimating cross sections when adding two-channel cross sections of the same molecular symmetry (Butler \& Dalgarno 1980b; Kingdon 1995). For singly-ionized atoms, CT usually cannot be treated in the LZ approximation, and hence we utilize the Demkov approximation to determine rate coefficients for those systems.

LZ calculations are generally accurate to within a factor of three for systems (and final channels) with large rate coefficients $\left(\gtrsim 10^{-9} \mathrm{~cm}^{3} \mathrm{~s}^{-1}\right.$; Butler \& Dalgarno 1980b; Kingdon \& Ferland 1996), but can be less accurate when the rate coefficient is small (though in that case, CT often is much less important than radiative and dielectronic recombination). The quantum mechanical molecular-orbital close-coupling (QMOCC) approach (e.g., Kimura \& Lane 1989; Zygelman et al. 1992; Wang et al. 2004) is the most accurate theoretical method for determining CT rate coefficients at the temperatures of interest. However, given the time- and computationally-intensive nature of such calculations, we have chosen to first render LZ calculations for these systems. We will test the sensitivity of abundance determinations to uncertainties in the CT rate coefficients (assumed to be roughly a factor of three) through numerical simulations of ionized nebulae. Those systems which are most critical for ionization equilibrium solutions are targets for further investigation with QMOCC methods.

The structure of this paper is as follows. In Sect. 2, we discuss the details and methodology of our calculations. We present the resulting total and final-state resolved CT rate coefficients in Sect. 3, and summarize our investigation in Sect. 4.

\section{Calculations and methodology}

\subsection{Multi-channel Landau Zener calculations}

For projectiles with charge $q=2-5$, we compute CT rate coefficients in the multi-channel Landau-Zener (MCLZ) approximation, based on the formalism of Butler \& Dalgarno (1980b) and Janev et al. (1983) with the low-energy modifications of Chang \& Pritchard (1979).

While CT is a quasi-molecular problem, the basic details of the interaction can be understood from consideration of the initial channel (in which both the projectile $\mathrm{X}^{q+}$ and target $\mathrm{H}$ are assumed to be in their ground states), the final channels (which can include ground and excited states of $\left.\mathrm{X}^{(q-1)+}\right)$, and the interaction between them. As the atoms approach each other, if the ionization potential of $\mathrm{X}^{(q-1)+}$ exceeds that of $\mathrm{H}$ (as is the case for $q=2-5$ for all elements we consider), CT is exoergic and the diabatic potential curves can cross at an internuclear distance $R_{\mathrm{x}}$. The CT reaction is usually driven by a radial coupling that connects initial and final channels corresponding to the same molecular symmetry of $\mathrm{XH}^{q+}$. Adiabatic potential curves of molecular states with the same symmetry cannot cross, and $R_{\mathrm{X}}$ corresponds to the radius at which the adiabatic potentials most closely approach (the "avoided crossing" distance).

In the LZ approximation, charge exchange is assumed to be localized at the internuclear distance $R_{\mathrm{x}}$, with the probability of exchange given by $2 p(1-p)$, where $p=\mathrm{e}^{-w}$ and

$w=\frac{\pi^{2}\left[\Delta U\left(R_{\mathrm{X}}\right)\right]^{2}}{h v\left[\frac{\mathrm{d}}{\mathrm{d} r}\left(H_{11}-H_{22}\right)\right]_{R_{\mathrm{X}}}}$.

In this equation, $v$ is the relative radial velocity of the projectile and target, $H_{11}$ and $H_{22}$ are the incoming and outgoing diabatic potentials, and $\Delta U\left(R_{\mathrm{x}}\right)$ is the separation of the adiabatic potentials at $R_{\mathrm{x}}$. The cross section at energy $E$ is

$\sigma(E)=4 \pi R_{\mathrm{x}}^{2} p_{0}(1+\lambda) \int_{1}^{\infty}\left(1-\mathrm{e}^{-w x}\right) \mathrm{e}^{-w x} x^{-3} \mathrm{~d} x$,

where $\lambda=\left[H_{11}(\infty)-H_{11}\left(R_{\mathrm{x}}\right)\right] / E_{i}$ (Butler \& Dalgarno 1980b). The approach probability $p_{0}$ is determined from the orbital and spin angular momenta of the initial and final states, which can form $g_{i f}=\left(2 L_{i}+1\right)\left(2 S_{i}+1\right)\left(2 L_{f}+1\right)\left(2 S_{f}+1\right)$ molecular states, and the spin $S$ and orbital angular momentum projection quantum number $\Lambda^{\prime}$ of the molecular state (Herzberg 1952; Dalgarno et al. 1990):

$p_{0}=(2 S+1)\left(2-\delta_{0, \Lambda^{\prime}}\right) / g_{i f}$.

The above describes a two-channel LZ interaction. In MCLZ calculations, several capture channels are considered simultaneously, each with a unique value of $R_{\mathrm{x}}$, as the projectile approaches the target. In this case, for $N$ final channels the probability for capture into a state $n$ is given by (Janev et al. 1983)

$$
\begin{aligned}
P_{n}= & p_{1} p_{2} \ldots p_{n}\left(1-p_{n}\right)\left[1+\left(p_{n+1} p_{n+2} \ldots p_{N}\right)^{2}\right. \\
& +\left(p_{n+1} p_{n+2} \ldots p_{N-1}\right)^{2}\left(1-p_{N}\right)^{2}+\ldots \\
& \left.+p_{n+1}^{2}\left(1-p_{n+2}\right)^{2}+\left(1-p_{n+1}\right)^{2}\right]
\end{aligned}
$$

LZ calculations require estimates of the incoming and outgoing potentials, the avoided crossing distance $R_{\mathrm{x}}$, and the energy separation of the adiabatic potentials at $R_{\mathrm{x}}$. We use approximations for these quantities from Butler \& Dalgarno (1980b) as follows. In atomic units ${ }^{1}$, the incoming potential is

$H_{11}(R)=\frac{-\alpha_{\mathrm{H}} q^{2}}{2 R^{4}}+A q \mathrm{e}^{-(0.8+\zeta) R}$,

where $\alpha_{\mathrm{H}}$ is the polarizability of $\mathrm{H}, R$ the internuclear distance, and $\zeta$ is the exponent of a single orbital wavefunction $(-1.0$ for $\mathrm{H}$ targets). Typically set to $25, A$ was reduced in some cases to yield physically reasonable behavior in the potential, which occasionally was spurious in the repulsive core (this generally only occurs when $R_{\mathrm{x}} \lesssim 4.5-5 a_{0}$, in which case the rate coefficient is usually small at photoionized plasma temperatures). The outgoing potential is given by

$H_{22}(R)=\frac{(q-1)}{R}-\Delta E$,

where $\Delta E=\operatorname{IP}\left(\mathrm{X}^{q+}\right)-\mathrm{IP}(\mathrm{H})-E_{\text {exc }}$ is the energy defect, IP is the ionization potential of the indicated system, and $E_{\text {exc }}$ is the excitation energy of the final $\mathrm{X}^{(q-1)+}$ state.

The avoided crossing distances $R_{\mathrm{x}}$ are determined by equating Eqs. (6) and (7). In Fig. 1, we illustrate the diabatic potentials and $R_{\mathrm{x}}$ values for the final channels of the case $\mathrm{X}^{q+}=\mathrm{Kr}^{3+}$. Note that we ignore fine structure in our calculations.

${ }^{1}$ All quantities in this paper are in atomic units unless otherwise noted. 
N. C. Sterling and P. C. Stancil: Atomic data for neutron-capture elements. III.

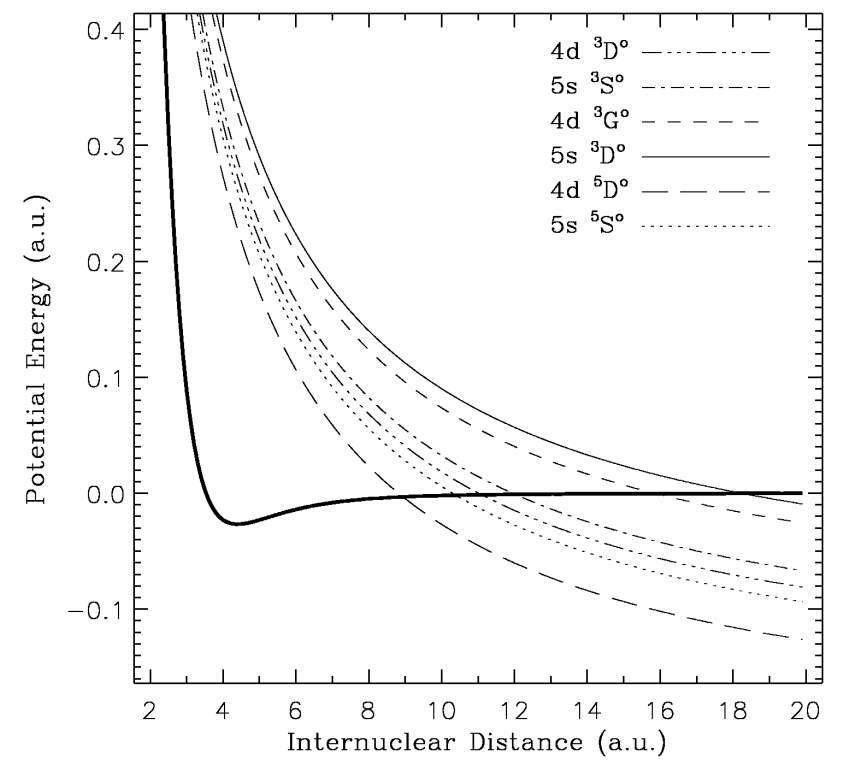

Fig. 1. Incoming diabatic potential $H_{11}$ for $\mathrm{Kr}^{3+}+\mathrm{H}$ (thick solid line), and diabatic potentials $H_{22}$ for six exit channels of $\mathrm{Kr}^{2+}+\mathrm{H}^{+}+\Delta E$ corresponding to ${ }^{3} \Sigma^{-}$and ${ }^{5} \Sigma^{-}$molecular symmetries (all listed states have an $[\mathrm{Ar}] 4 \mathrm{~s}^{2} 4 \mathrm{p}^{3}$ core). The intersections of the incoming and outgoing potentials correspond to the avoided crossing distances $R_{\mathrm{x}}$ for each exit channel. The potential energies are shifted by taking $H_{11}(\infty)=0$.

The final piece of necessary information, $\Delta U\left(R_{\mathrm{x}}\right)$, is perhaps the greatest uncertainty in LZ calculations, as the formula provided by Butler \& Dalgarno (1980b) is an empirical fit to a small number of quantal calculations. The value of $\Delta U\left(R_{\mathrm{x}}\right)$ depends on whether the electron capture process is type I (the $\mathrm{H} 1 s$ electron is transferred without affecting the core electrons of the projectile) or type II (electron capture is accompanied by the excitation of a core electron). The formulae of Butler \& Dalgarno (1980b) for these two scenarios are:

$$
\begin{aligned}
\Delta U_{\mathrm{I}} & =27.21 R_{\mathrm{x}}^{2} \mathrm{e}^{-\beta R_{\mathrm{x}}} \\
\Delta U_{\mathrm{II}} & =0.5 \mathrm{e}^{-0.4 R_{\mathrm{x}}} \Delta U_{\mathrm{I}},
\end{aligned}
$$

where $\beta=2 \mathrm{IP}(\mathrm{H})=1.0$ in atomic units. The potential for type II reactions is reduced relative to their type I counterparts due to the rearrangement of the core electrons.

Once the quantities $H_{11}, H_{22}, R_{\mathrm{x}}$, and $\Delta U\left(R_{\mathrm{x}}\right)$ are determined, then the cross section for each final channel can be determined from Eq. (3), as illustrated for $\mathrm{X}^{q+}=\mathrm{Kr}^{3+}$ in Fig. 2. These are integrated over a Maxwellian velocity distribution to derive final-state resolved rate coefficients.

\subsection{Demkov calculations}

In the case of singly-charged ions $(q=1$ in Eq. (1)), the LZ approximation cannot be applied since the final channels do not have Coulomb potentials (hence there are no avoided crossings). The reaction proceeds through radial coupling connecting molecular states with the same symmetry as in the LZ calculations, but the coupling is not localized and can be represented with an exponential form. This type of interaction can be described by the Demkov approximation (Demkov 1964; Olson 1972; Swartz 1994).

The reaction is assumed to occur at an interaction distance that we label $R_{\mathrm{x}}$ due to its analogous nature to the avoided

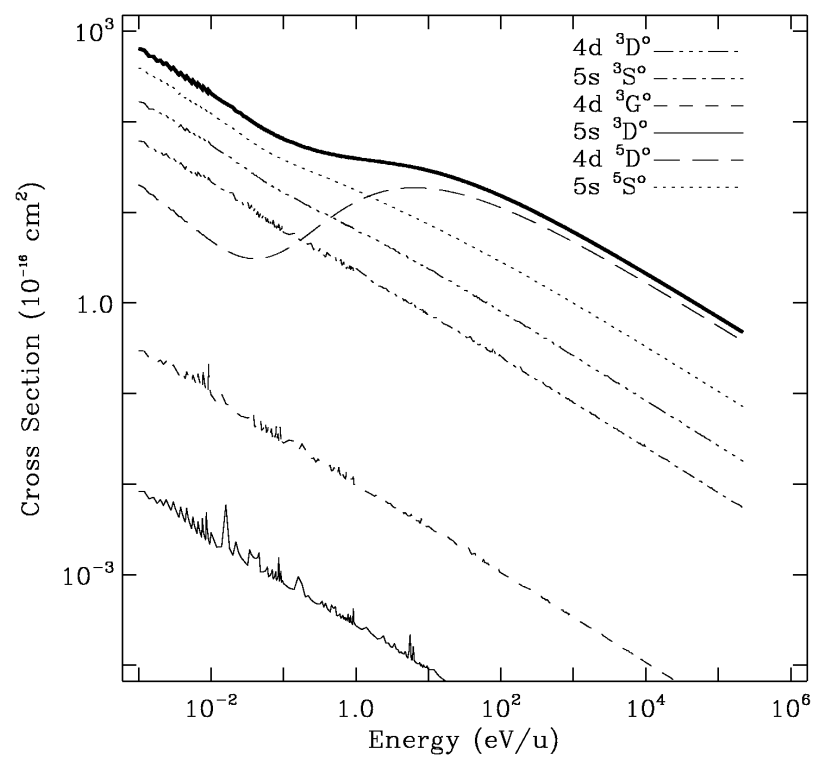

Fig. 2. Total (thick solid curve) and final-state resolved cross sections for $\mathrm{CT}$ recombination of $\mathrm{Kr}^{3+}$. Energies are given in $\mathrm{eV} / \mathrm{u}$, where $\mathrm{u}$ is the atomic mass unit.

crossing distance in LZ calculations. $R_{\mathrm{x}}$ is given by equating $\Delta U(R)$ with (Swartz 1994)

$\Delta H(R)=H_{11}\left(\mathrm{X}^{+}+\mathrm{H}\right)-H_{22}\left(\mathrm{X}+\mathrm{H}^{+}\right)-\Delta E$.

For the potential $H_{11}\left(\mathrm{X}+\mathrm{H}^{+}\right)$, the polarizability term (see Eq. (6)) should include a quadrupole term in addition to the dipole polarizability if $\mathrm{X}$ is not an $S$ ground term (Gentry \& Giese 1977):

$\frac{\alpha_{\mathrm{d}}}{2 R^{4}} \rightarrow \frac{\alpha_{\mathrm{d}}}{2 R^{4}} \pm \frac{Q}{2 R^{3}}$.

To determine $\Delta H(R)$ for each element, static average electric dipole polarizabilities were taken from Lide (2002) and quadrupole matrix elements from Tables 2 and 3 of Froese Fischer (1977).

The transition probability for the reaction is $p=\mathrm{e}^{-w}$ with (Demkov 1964; Swartz 1994)

$w=\left[1+\exp \left(\frac{2 \pi^{2} \Delta U\left(R_{\mathrm{x}}\right)}{h \alpha v}\right)\right]^{-1}$,

where $h$ is Planck's constant and $\alpha$ is the polarizability of the neutral species X. Defining

$\delta=\frac{\pi^{2} \Delta U\left(R_{\mathrm{x}}\right)}{h \alpha v}$,

the cross section at energy $E$ is

$\sigma(E)=\pi R_{\mathrm{x}}^{2} \int_{1}^{\infty} \frac{1}{x^{3}}\left(\frac{4 \mathrm{e}^{-\delta x}}{\left(1+\mathrm{e}^{\delta x}\right)^{2}}\right) \mathrm{d} x$.

We compute two-channel cross sections, which can be added to determine the total cross section. While this addition can lead to overestimated total cross sections (see Sect. 2.1), only Se and $\mathrm{Br}$ have more than one exoergic final channel, and their cross sections are typically small in the relevant temperature range. As for the MCLZ calculations, the cross sections are integrated over a Maxwellian velocity distribution to derive rate coefficients. 


\section{Results}

In Table 1, we provide information for the exit channels of our MCLZ and Demkov calculations, including energy defects $\triangle \mathrm{E}$, avoided crossing distances (or interaction distances, for singlycharged ions) $R_{\mathrm{x}}$, and the energy separation $\Delta U$ of the adiabatic curves at $R_{\mathrm{X}}$. These parameters were used to compute the $\mathrm{CT}$ cross section for each final channel. In addition, we list the molecular symmetries common to the initial and final channels and whether the reaction is type I or type II. When a final state can connect to the initial state via more than one molecular symmetry, the cross sections for each symmetry were added to determine the state-specific cross section. The state-resolved cross sections were added to determine the total CT cross section, and rate coefficients determined by integrating over a Maxwellian velocity distribution.

The total CT recombination rate coefficients of each ion are given in Table 2 as functions of temperature, and are illustrated in Fig. 3. We note that for $q=5$, highly-excited Rydberg states can be favorable exit channels, but the reported level energies in NIST for $\mathrm{X}^{4+}$ ions are incomplete at those energies. Thus, CT into those highly-excited states could not be computed, and as a result the rate coefficients for $q=5$ are lower limits. As seen in Fig. 3, the computed $q=5$ rate coefficients are smaller than those for $q=4$ with the exception of $\mathrm{Ge}^{5+}$. Since CT rate coefficients generally increase with charge for $q \geq 3$, we recommend that $q=4 \mathrm{CT}$ rate coefficients be used as rough estimates for $\mathrm{Se}$, $\mathrm{Br}, \mathrm{Kr}, \mathrm{Rb}$, and $\mathrm{Xe} q \geq 5$ ions.

When the radial coupling CT rate coefficient from our MCLZ and Demkov calculations is sufficiently small, CT can proceed via other coupling mechanisms. Two such examples are radiative $\mathrm{CT}$ and spin-orbit (SO) coupling. For radiative $\mathrm{CT}$, the canonical rate coefficient is taken to be $10^{-14} \mathrm{~cm}^{3} \mathrm{~s}^{-1}$ (Butler et al. 1977). This value is uncertain by at least an order of magnitude (Butler et al. 1977; Stancil \& Zygelman 1996), but given the small rate coefficient, CT is unlikely to be an important recombination mechanism compared to radiative or dielectronic recombination when radiative $\mathrm{CT}$ dominates radial coupling. The rate coefficients listed in the tables are the maximum of the radially-coupled and radiative CT rate coefficients (thus, all values of 1.00E-14 indicate that radiative CT dominates radiallycoupled CT). For some exit channels, radiative CT dominates radial coupling at all temperatures from $10^{2}-10^{6} \mathrm{~K}$. States with such small rate coefficients tend to be associated with small or large avoided crossing distances $\left(R_{\mathrm{x}} \lesssim 5 a_{0}, R_{\mathrm{x}} \gtrsim 15-20 a_{0}\right)$. For some singly- and doubly-charged ions, the only exothermic exit channel(s) has a very small rate coefficient dominated by radiative $\mathrm{CT}$.

Spin-orbit coupling is not as well understood as the radial or radiative mechanisms. Rate coefficients for SO-coupled $\mathrm{CT}$ reactions can range from $10^{-17}-10^{-12} \mathrm{~cm}^{3} \mathrm{~s}^{-1}$ (Butler \& Dalgarno 1979, 1980a; Pradhan \& Dalgarno 1994; Stancil 2001), though detailed calculations are very few and to our knowledge exist only for singly-charged ions. Because of this, we consider SO coupling only for CT recombination of singly-charged ions and CT ionization of neutral species. For small radial coupling CT rate coefficients $\left(\leq 10^{-12} \mathrm{~cm}^{3} \mathrm{~s}^{-1}\right)$, we adopt $10^{-12} \mathrm{~cm}^{3} \mathrm{~s}^{-1}$, which is the largest computed rate coefficient for SO coupling (Pradhan \& Dalgarno 1994), as an upper limit to the rate coefficient. This estimate provides a much smaller, but more robust upper limit than estimates based on the Langevin model (Stancil 2001).

However, SO coupling is not relevant for all of the singlyionized species we consider, since it only connects initial and
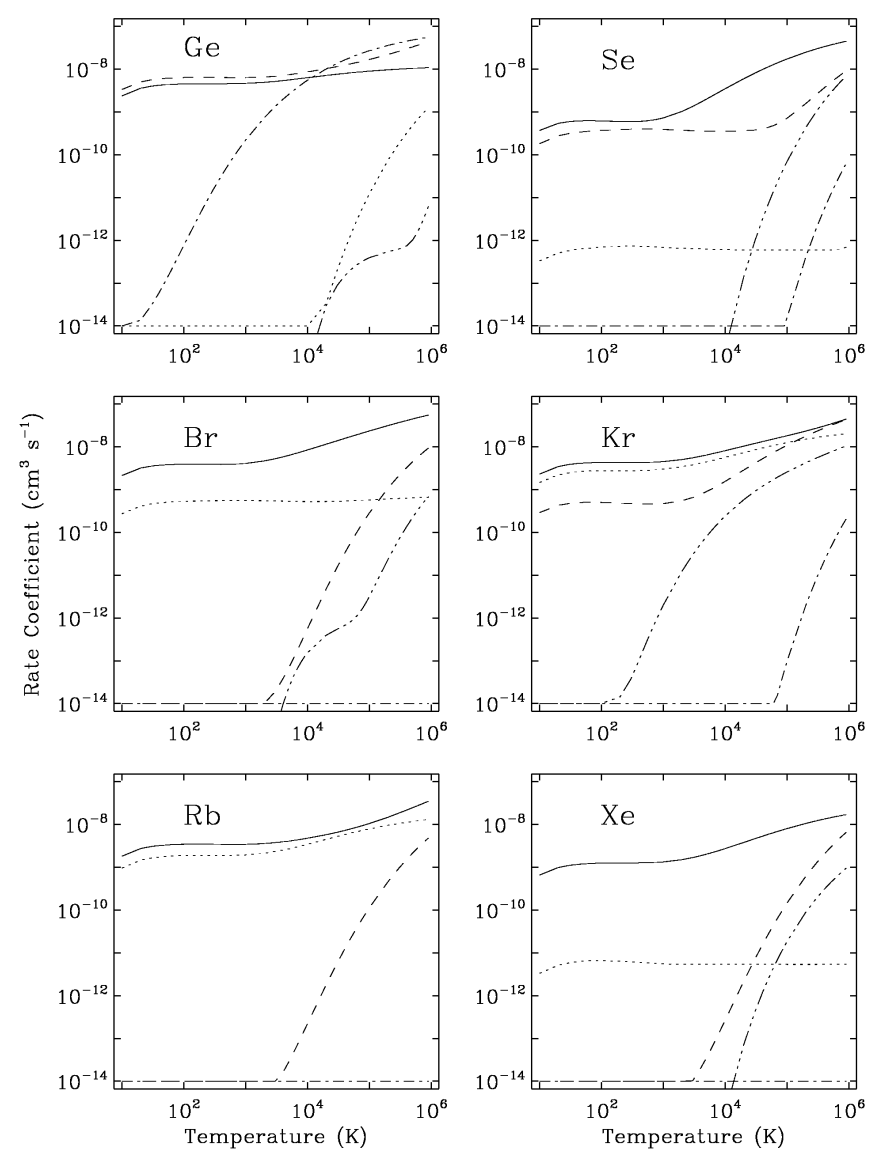

Fig. 3. Total rate coefficients for CT recombination of $\mathrm{X}^{+}$(dash-dotdot-dot-dash curves), $\mathrm{X}^{2+}$ (dash-dot-dash curves), $\mathrm{X}^{3+}$ (dotted curves), $\mathrm{X}^{4+}$ (solid curves), and $\mathrm{X}^{5+}$ (dashed curves), where $\mathrm{X}$ is the element indicated in each panel. The $q=5$ rate coefficients are lower limits due to incomplete experimental energy level measurements. Note that the $\mathrm{Rb}^{+}$rate coefficient is below $10^{-14} \mathrm{~cm}^{3} \mathrm{~s}^{-1}$ over the plotted temperature range.

final states of different molecular symmetries for which radial and rotational coupling are forbidden. For CT recombination of $\mathrm{Rb}^{+}$(and the reverse reaction), the entrance and exit channels correspond to ${ }^{2} \Sigma^{+}$molecular states, and SO coupling therefore plays no role. In the case of $\mathrm{Kr}^{+}$and $\mathrm{Xe}^{+}$, for which there is only one exothermic exit channel, the diabatic potentials for the triplet exit channels do not cross the potential curve of the singlet entrance channel, and hence SO coupling is unlikely to be important for these reactions. We therefore adopt the SO coupling upper limit to the rate coefficients only for singly-ionized $\mathrm{Ge}, \mathrm{Se}$, and $\mathrm{Br}$.

CT ionization is exothermic for the neutrals of the elements we consider, with the exception of $\mathrm{Kr}$. We provide rate coefficients for these systems in Table 3, and depict them in Fig. 4. As for CT recombination, we adopt the largest of the rate coefficients associated with the radial, radiative, and (when important) SO mechanisms.

The CT recombination rate coefficients for singly-ionized species were computed from the CT ionization rate coefficients via detailed balance:

$\alpha_{\text {rec }}=\alpha_{\text {ion }} \frac{p_{0, \text { rec }}}{p_{0, \text { ion }}} \exp \left(\frac{\Delta E_{\infty}}{k_{\mathrm{B}} T}\right)$,

where $\alpha_{\text {rec,ion }}$ are the rate coefficients for CT recombination and ionization, respectively, $p_{0 \text {,rec,ion }}$ are the approach probabilities 
N. C. Sterling and P. C. Stancil: Atomic data for neutron-capture elements. III.

Table 1. Details of MCLZ and Demkov calculations.

\begin{tabular}{|c|c|c|c|c|c|}
\hline $\begin{array}{l}\text { Exit } \\
\text { Channels }^{a}\end{array}$ & $\begin{array}{l}\Delta E \\
\text { (a.u.) }\end{array}$ & $\begin{array}{c}R_{\mathrm{x}} \\
\text { (a.u.) }\end{array}$ & $\begin{array}{c}\Delta U\left(R_{\mathrm{x}}\right) \\
\text { (a.u.) }\end{array}$ & $\begin{array}{c}\text { Molecular } \\
\text { Symmetries }^{b}\end{array}$ & Type \\
\hline \multicolumn{6}{|c|}{$\mathrm{Ge}^{0}\left({ }^{3} \mathrm{P}\right)+\mathrm{H}^{+}\left({ }^{1} \mathrm{~S}\right) \rightleftharpoons \mathrm{Ge}^{+}+\mathrm{H}\left({ }^{2} \mathrm{~S}\right)+\Delta E \quad\left({ }^{3} \Sigma^{-},{ }^{3} \Pi\right)$} \\
\hline $4 s^{2} 4 p^{2} \mathrm{P}^{\circ}$ & 0.2041 & 7.258 & 0.2089 & ${ }^{3} \Sigma^{-}$ & $\mathrm{I}$ \\
\hline $4 s^{2} 4 p^{2} \mathrm{P}^{\circ}$ & 0.2041 & 7.406 & 0.1943 & ${ }^{3} \Pi$ & I \\
\hline \multicolumn{6}{|c|}{$\mathrm{Ge}^{2+}\left({ }^{1} \mathrm{~S}\right)+\mathrm{H}\left({ }^{2} \mathrm{~S}\right) \rightarrow \mathrm{Ge}^{+}+\mathrm{H}^{+}\left({ }^{1} \mathrm{~S}\right)+\Delta E \quad\left({ }^{2} \Sigma^{+}\right)$} \\
\hline $4 s^{2} 4 p^{2} \mathrm{P}^{\circ}$ & 0.0805 & 12.48 & $5.922 \mathrm{E}-03$ & ${ }^{2} \Sigma^{+}$ & $\mathrm{I}$ \\
\hline \multicolumn{6}{|c|}{$\mathrm{Ge}^{3+}\left({ }^{2} \mathrm{~S}\right)+\mathrm{H}\left({ }^{2} \mathrm{~S}\right) \rightarrow \mathrm{Ge}^{2+}+\mathrm{H}^{+}\left({ }^{1} \mathrm{~S}\right)+\Delta E \quad\left({ }^{1} \Sigma^{+},{ }^{3} \Sigma^{+}\right)$} \\
\hline $4 \mathrm{~s} 4 \mathrm{p}^{3} \mathrm{P}^{\circ}$ & 0.4633 & 4.648 & 0.2070 & ${ }^{3} \Sigma^{+}$ & I \\
\hline $4 \mathrm{~s} 4 \mathrm{p}^{1} \mathrm{P}^{\circ}$ & 0.3333 & 6.240 & 7.592E-02 & ${ }^{1} \Sigma^{+}$ & $\mathrm{I}$ \\
\hline \multicolumn{6}{|c|}{$\mathrm{Ge}^{4+}\left({ }^{1} \mathrm{~S}\right)+\mathrm{H}\left({ }^{2} \mathrm{~S}\right) \rightarrow \mathrm{Ge}^{3+}+\mathrm{H}^{+}\left({ }^{1} \mathrm{~S}\right)+\Delta E \quad\left({ }^{2} \Sigma^{+}\right)$} \\
\hline $4 p^{2} \mathrm{P}^{\circ}$ & 0.8013 & 4.138 & 0.2732 & ${ }^{2} \Sigma^{+}$ & $\mathrm{I}$ \\
\hline $4 d^{2} \mathrm{D}$ & 0.3111 & 9.768 & $5.463 \mathrm{E}-03$ & ${ }^{2} \Sigma^{+}$ & I \\
\hline $5 s^{2} S$ & 0.2724 & 11.11 & $1.847 \mathrm{E}-03$ & ${ }^{2} \Sigma^{+}$ & I \\
\hline \multicolumn{6}{|c|}{$\mathrm{Ge}^{5+}\left({ }^{2} \mathrm{D}\right)+\mathrm{H}\left({ }^{2} \mathrm{~S}\right) \rightarrow \mathrm{Ge}^{4+}+\mathrm{H}^{+}\left({ }^{1} \mathrm{~S}\right)+\Delta E \quad\left({ }^{1} \Sigma^{+},{ }^{1} \Pi,{ }^{1} \Delta,{ }^{3} \Sigma^{+},{ }^{3} \Pi,{ }^{3} \Delta\right)$} \\
\hline $3 d^{9} 4 d^{3} S$ & 0.7481 & 5.723 & 0.1071 & ${ }^{3} \Sigma^{+}$ & I \\
\hline $3 d^{9} 4 d^{1} P$ & 0.7219 & 5.901 & $9.530 \mathrm{E}-02$ & ${ }^{1} \Pi$ & I \\
\hline $3 d^{9} 4 d^{3} G$ & 0.7202 & 5.913 & $9.454 \mathrm{E}-02$ & ${ }^{3} \Sigma^{+},{ }^{3} \Pi,{ }^{3} \Delta$ & $\mathrm{I}$ \\
\hline $3 d^{9} 4 d^{3} P$ & 0.7161 & 5.942 & $9.274 \mathrm{E}-02$ & ${ }^{3} \Pi$ & I \\
\hline $3 d^{9} 4 d^{3} D$ & 0.7095 & 5.990 & $8.983 \mathrm{E}-02$ & ${ }^{3} \Sigma^{+},{ }^{3} \Pi,{ }^{3} \Delta$ & I \\
\hline $3 d^{9} 4 d^{1} F$ & 0.6993 & 6.065 & $8.544 \mathrm{E}-02$ & ${ }^{1} \Pi,{ }^{1} \Delta$ & I \\
\hline $3 d^{9} 4 d^{1} D$ & 0.6905 & 6.132 & $8.168 \mathrm{E}-02$ & ${ }^{1} \Sigma^{+},{ }^{1} \Pi,{ }^{1} \Delta$ & I \\
\hline $3 d^{9} 4 d^{3} F$ & 0.6903 & 6.134 & $8.157 \mathrm{E}-02$ & ${ }^{3} \Pi,{ }^{3} \Delta$ & I \\
\hline $3 d^{9} 5 s^{3} D$ & 0.5888 & 7.062 & $4.274 \mathrm{E}-02$ & ${ }^{3} \Sigma^{+},{ }^{3} \Pi,{ }^{3} \Delta$ & I \\
\hline $3 d^{9} 4 d^{1} S$ & 0.5763 & 7.200 & $3.870 \mathrm{E}-02$ & ${ }^{1} \Sigma^{+}$ & I \\
\hline $3 d^{9} 5 s^{3} P$ & 0.5677 & 7.299 & $3.603 \mathrm{E}-02$ & ${ }^{3} \Pi$ & I \\
\hline $3 d^{9} 5 p^{3} \mathrm{P}^{\circ}$ & 0.4370 & 9.313 & $7.827 \mathrm{E}-03$ & ${ }^{3} \Sigma^{+},{ }^{3} \Pi$ & I \\
\hline $3 d^{9} 5 p^{3} F^{\circ}$ & 0.4313 & 9.430 & $7.139 \mathrm{E}-03$ & ${ }^{3} \Sigma^{+},{ }^{3} \Pi,{ }^{3} \Delta$ & I \\
\hline $3 d^{9} 5 p^{1} D^{\circ}$ & 0.42 & 9.506 & $6.724 \mathrm{E}-03$ & ${ }^{1} \Pi,{ }^{1} \Delta$ & I \\
\hline $3 d^{9} 5 p^{3} D^{\circ}$ & 0.4149 & 9.786 & $5.385 \mathrm{E}-03$ & ${ }^{3} \Pi,{ }^{3} \Delta$ & I \\
\hline $3 d^{9} 5 p^{1} P^{\circ}$ & 0.4136 & 9.815 & $5.262 \mathrm{E}-03$ & ${ }^{1} \Sigma^{+},{ }^{1} \Pi$ & I \\
\hline $3 d^{9} 5 p^{1} F^{\circ}$ & 0.4107 & 9.882 & 4.989E-03 & ${ }^{1} \Sigma^{+},{ }^{1} \Pi,{ }^{1} \Delta$ & I \\
\hline \multicolumn{6}{|c|}{$\mathrm{Se}^{0}\left({ }^{3} \mathrm{P}\right)+\mathrm{H}^{+}\left({ }^{1} \mathrm{~S}\right) \rightleftharpoons \mathrm{Se}^{+}+\mathrm{H}\left({ }^{2} \mathrm{~S}\right)+\Delta E \quad\left({ }^{3} \Sigma^{-},{ }^{3} \Pi\right)$} \\
\hline $4 s^{2} 4 p^{34} S^{\circ}$ & 0.1413 & 7.006 & 0.1304 & ${ }^{3} \Sigma^{-}$ & I \\
\hline $4 s^{2} 4 p^{32} D^{\circ}$ & 0.0796 & 7.860 & $7.958 \mathrm{E}-02$ & ${ }^{3} \Pi$ & I \\
\hline $4 s^{2} 4 p^{32} D^{\circ}$ & 0.0796 & 8.020 & 7.240E-02 & ${ }^{3} \Sigma^{-}$ & I \\
\hline $4 \mathrm{~s}^{2} 4 \mathrm{p}^{32} \mathrm{P}^{\circ}$ & 0.0338 & 9.251 & $3.396 \mathrm{E}-02$ & ${ }^{3} \Pi$ & I \\
\hline \multicolumn{6}{|c|}{$\mathrm{Se}^{2+}\left({ }^{3} \mathrm{P}\right)+\mathrm{H}\left({ }^{2} \mathrm{~S}\right) \rightarrow \mathrm{Se}^{+}+\mathrm{H}^{+}\left({ }^{1} \mathrm{~S}\right)+\Delta E \quad\left({ }^{2} \Sigma^{-},{ }^{2} \Pi,{ }^{4} \Sigma^{-},{ }^{4} \Pi\right)$} \\
\hline $4 \mathrm{~s}^{2} 4 \mathrm{p}^{32} \mathrm{P}^{\circ}$ & 0.1714 & 6.040 & $8.688 \mathrm{E}-02$ & ${ }^{2} \Pi$ & I \\
\hline \multicolumn{6}{|c|}{$\mathrm{Se}^{3+}\left({ }^{2} \mathrm{P}^{\circ}\right)+\mathrm{H}\left({ }^{2} \mathrm{~S}\right) \rightarrow \mathrm{Se}^{2+}+\mathrm{H}^{+}\left({ }^{1} \mathrm{~S}\right)+\Delta E \quad\left({ }^{1} \Sigma^{+},{ }^{1} \Pi,{ }^{3} \Sigma^{+},{ }^{3} \Pi\right)$} \\
\hline $4 s^{2} 4 p^{2}{ }^{1} S$ & 0.5034 & 4.256 & 0.2568 & ${ }^{1} \Sigma^{+}$ & I \\
\hline $4 s 4 p^{3}{ }^{3} D^{\circ}$ & 0.2037 & 9.921 & $4.588 \mathrm{E}-05$ & ${ }^{3} \Pi$ & II \\
\hline \multicolumn{6}{|c|}{$\mathrm{Se}^{4+}\left({ }^{1} \mathrm{~S}\right)+\mathrm{H}\left({ }^{2} \mathrm{~S}\right) \rightarrow \mathrm{Se}^{3+}+\mathrm{H}^{+}\left({ }^{1} \mathrm{~S}\right)+\Delta E \quad\left({ }^{2} \Sigma^{+}\right)$} \\
\hline $4 s 4 p^{22} D$ & 0.6023 & 5.328 & $8.177 \mathrm{E}-03$ & ${ }^{2} \Sigma^{+}$ & II \\
\hline $4 s 4 p^{2}{ }^{2} s$ & 0.4917 & 6.374 & $2.706 \mathrm{E}-03$ & ${ }^{2} \Sigma^{+}$ & II \\
\hline $4 s^{2} 4 d^{2} D$ & 0.3793 & 8.088 & $2.010 \mathrm{E}-02$ & ${ }^{2} \Sigma^{+}$ & I \\
\hline $4 s^{2} 5 s^{2} S$ & 0.3620 & 8.452 & $1.525 \mathrm{E}-02$ & ${ }^{2} \Sigma^{+}$ & I \\
\hline $4 s^{2} 5 p^{2} \mathrm{P}^{\circ}$ & 0.2095 & 14.38 & $1.178 \mathrm{E}-04$ & ${ }^{2} \Sigma^{+}$ & I \\
\hline \multicolumn{6}{|c|}{$\mathrm{Se}^{5+}\left({ }^{2} \mathrm{~S}\right)+\mathrm{H}\left({ }^{2} \mathrm{~S}\right) \rightarrow \mathrm{Se}^{4+}+\mathrm{H}^{+}\left({ }^{1} \mathrm{~S}\right)+\Delta E \quad\left({ }^{1} \Sigma^{+},{ }^{3} \Sigma^{+}\right)$} \\
\hline $4 \mathrm{~s} 4 \mathrm{~d}^{1} \mathrm{D}$ & 1.0394 & 4.342 & 0.2453 & ${ }^{1} \Sigma^{+}$ & I \\
\hline $4 s 4 d{ }^{3} \mathrm{D}$ & 0.8359 & 5.209 & 0.1483 & ${ }^{3} \Sigma^{+}$ & I \\
\hline $4 s 4 d{ }^{3} \mathrm{D}$ & 0.7012 & 6.051 & $8.625 \mathrm{E}-02$ & ${ }^{3} \Sigma^{+}$ & I \\
\hline $4 \mathrm{~s} 4 \mathrm{f}^{1} \mathrm{~F}^{\circ}$ & 0.3639 & 11.11 & $1.854 \mathrm{E}-03$ & ${ }^{1} \Sigma^{+}$ & I \\
\hline \multicolumn{6}{|c|}{$\mathrm{Br}^{0}\left({ }^{2} \mathrm{P}^{\circ}\right)+\mathrm{H}^{+}\left({ }^{1} \mathrm{~S}\right) \rightleftharpoons \mathrm{Br}^{+}+\mathrm{H}\left({ }^{2} \mathrm{~S}\right)+\Delta E \quad\left({ }^{2} \Sigma^{+},{ }^{2} \Pi\right)$} \\
\hline $4 s^{2} 4 p^{43} \mathrm{P}$ & 0.0589 & 7.241 & $6.159 \mathrm{E}-02$ & ${ }^{2} \Pi$ & I \\
\hline $4 s^{2} 4 p^{41} D$ & 0.0105 & 9.612 & $1.191 \mathrm{E}-02$ & ${ }^{2} \Pi$ & I \\
\hline $4 s^{2} 4 p^{4}{ }^{1} D$ & 0.0105 & 10.05 & $8.681 \mathrm{E}-03$ & ${ }^{2} \Sigma^{+}$ & $\mathrm{I}$ \\
\hline \multicolumn{6}{|c|}{$\mathrm{Br}^{2+}\left({ }^{4} \mathrm{~S}^{\circ}\right)+\mathrm{H}\left({ }^{2} \mathrm{~S}\right) \rightarrow \mathrm{Br}^{+}+\mathrm{H}^{+}\left({ }^{1} \mathrm{~S}\right)+\Delta E \quad\left({ }^{3} \Sigma^{-},{ }^{5} \Sigma^{-}\right)$} \\
\hline $4 s^{2} 4 p^{43} \mathrm{P}$ & 0.2947 & 3.576 & 0.3579 & ${ }^{3} \Sigma^{-}$ & I \\
\hline
\end{tabular}


Table 1. continued.

\begin{tabular}{|c|c|c|c|c|c|}
\hline $\begin{array}{l}\text { Exit } \\
\text { Channels }^{a}\end{array}$ & $\begin{array}{l}\Delta E \\
\text { (a.u.) }\end{array}$ & $\begin{array}{c}R_{\mathrm{x}} \\
\text { (a.u.) }\end{array}$ & $\begin{array}{c}\Delta U\left(R_{\mathrm{x}}\right) \\
\text { (a.u.) }\end{array}$ & $\begin{array}{c}\text { Molecular } \\
\text { Symmetries }^{b}\end{array}$ & Type \\
\hline \multicolumn{6}{|c|}{$\mathrm{Br}^{3+}\left({ }^{3} \mathrm{P}\right)+\mathrm{H}\left({ }^{2} \mathrm{~S}\right) \rightarrow \mathrm{Br}^{2+}+\mathrm{H}^{+}\left({ }^{1} \mathrm{~S}\right)+\Delta E$} \\
\hline $4 \mathrm{~s} 4 \mathrm{p}^{42} \mathrm{D}$ & 0.4068 & 5.219 & $9.140 \mathrm{E}-03$ & ${ }^{2} \Pi$ & II \\
\hline $4 s^{2} 4 p^{2} 5 s^{4} P$ & 0.1462 & 13.73 & $2.046 \mathrm{E}-04$ & ${ }^{4} \Sigma^{-},{ }^{4} \Pi$ & I \\
\hline $4 s^{2} 4 p^{2} 5 s^{2} P$ & 0.1346 & 14.91 & 7.473E-05 & ${ }^{2} \Sigma^{-},{ }^{2} \Pi$ & I \\
\hline \multicolumn{6}{|c|}{$\mathrm{Br}^{4+}\left({ }^{2} \mathrm{P}^{\circ}\right)+\mathrm{H}\left({ }^{2} \mathrm{~S}\right) \rightarrow \mathrm{Br}^{3+}+\mathrm{H}^{+}\left({ }^{1} \mathrm{~S}\right)+\Delta E \quad\left({ }^{1} \Sigma^{+},{ }^{1} \Pi,{ }^{3} \Sigma^{+},{ }^{3} \Pi\right)$} \\
\hline $4 \mathrm{~s} 4 \mathrm{p}^{33} \mathrm{D}^{\circ}$ & 0.5728 & 5.570 & $6.369 \mathrm{E}-03$ & ${ }^{3} \Pi$ & II \\
\hline $4 \mathrm{~s} 4 \mathrm{p}^{3}{ }^{3} \mathrm{P}^{\circ}$ & 0.5091 & 6.176 & $3.352 \mathrm{E}-03$ & ${ }^{3} \Sigma^{+},{ }^{3} \Pi$ & II \\
\hline $4 s 4 p^{3}{ }^{1} D^{\circ}$ & 0.5002 & 6.277 & $3.006 \mathrm{E}-03$ & ${ }^{1} \Pi$ & II \\
\hline $4 s^{2} 4 p 4 d^{3} F^{\circ}$ & 0.4212 & 7.336 & $3.507 \mathrm{E}-02$ & ${ }^{3} \Sigma^{+},{ }^{3} \Pi$ & I \\
\hline $4 s 4 p^{3}{ }^{1} \mathrm{P}^{\circ}$ & 0.4157 & 7.426 & $8.421 \mathrm{E}-04$ & ${ }^{1} \Sigma^{+},{ }^{1} \Pi$ & II \\
\hline $4 s^{2} 4 p 4 d^{3} D^{\circ}$ & 0.3800 & 8.074 & $2.031 \mathrm{E}-02$ & ${ }^{3} \Pi$ & I \\
\hline $4 s^{2} 4 p 4 d^{3} P^{\circ}$ & 0.3468 & 8.803 & $1.165 \mathrm{E}-02$ & ${ }^{3} \Sigma^{+},{ }^{3} \Pi$ & I \\
\hline $4 s^{2} 4 p 4 d^{1} F^{\circ}$ & 0.3448 & 8.852 & $1.121 \mathrm{E}-02$ & ${ }^{1} \Sigma^{+},{ }^{1} \Pi$ & I \\
\hline $4 s^{2} 4 p 4 d^{1} P^{\circ}$ & 0.3301 & 9.227 & $8.373 \mathrm{E}-03$ & ${ }^{1} \Sigma^{+},{ }^{1} \Pi$ & I \\
\hline $4 s^{2} 4 p 4 d^{1} D^{\circ}$ & 0.3245 & 9.380 & $7.426 \mathrm{E}-03$ & ${ }^{1} \Pi$ & I \\
\hline $4 s^{2} 4 p 5 s^{3} P^{\circ}$ & 0.3180 & 9.564 & $6.422 \mathrm{E}-03$ & ${ }^{3} \Sigma^{+},{ }^{3} \Pi$ & I \\
\hline $4 s^{2} 4 p 5 s^{1} P^{\circ}$ & 0.3035 & 10.04 & $4.526 \mathrm{E}-03$ & ${ }^{1} \Sigma^{+},{ }^{1} \Pi$ & I \\
\hline $4 s^{2} 4 p 5 p^{3} D$ & 0.1619 & 18.57 & $2.983 \mathrm{E}-06$ & ${ }^{3} \Sigma^{+},{ }^{3} \Pi$ & I \\
\hline \multicolumn{6}{|c|}{$\mathrm{Br}^{5+}\left({ }^{1} \mathrm{~S}\right)+\mathrm{H}\left({ }^{2} \mathrm{~S}\right) \rightarrow \mathrm{Br}^{4+}+\mathrm{H}^{+}\left({ }^{1} \mathrm{~S}\right)+\Delta E \quad\left({ }^{2} \Sigma^{+}\right)$} \\
\hline $4 s 4 p^{2}{ }^{2} \mathrm{D}$ & 1.1321 & 4.040 & $2.853 \mathrm{E}-02$ & $2 \Sigma^{+}$ & II \\
\hline $4 s^{2} 4 d^{2} D$ & 0.8360 & 5.209 & 0.1483 & ${ }^{2} \Sigma^{+}$ & I \\
\hline $4 s^{2} 5 s^{2} S$ & 0.7214 & 5.904 & $9.511 \mathrm{E}-02$ & ${ }^{2} \Sigma^{+}$ & I \\
\hline \multicolumn{6}{|c|}{$\mathrm{Kr}^{+}\left({ }^{2} \mathrm{P}^{\circ}\right)+\mathrm{H}\left({ }^{2} \mathrm{~S}\right) \rightleftharpoons \mathrm{Kr}+\mathrm{H}^{+}\left({ }^{1} \mathrm{~S}\right)+\Delta E \quad\left({ }^{1} \Sigma^{+},{ }^{1} \Pi,{ }^{3} \Sigma^{+},{ }^{3} \Pi\right)$} \\
\hline $4 s^{2} 4 p^{6}{ }^{1} S$ & 0.0147 & 8.394 & $1.595 \mathrm{E}-02$ & ${ }^{1} \Sigma^{+}$ & I \\
\hline \multicolumn{6}{|c|}{$\mathrm{Kr}^{2+}\left({ }^{3} \mathrm{P}\right)+\mathrm{H}\left({ }^{2} \mathrm{~S}\right) \rightarrow \mathrm{Kr}^{+}+\mathrm{H}^{+}\left({ }^{1} \mathrm{~S}\right)+\Delta E \quad\left({ }^{2} \Sigma^{-},{ }^{2} \Pi,{ }^{4} \Sigma^{-},{ }^{4} \Pi\right)$} \\
\hline $4 s^{2} 4 p^{5}{ }^{2} \mathrm{P}^{\circ}$ & 0.3873 & 2.677 & 0.4928 & ${ }^{2} \Pi$ & I \\
\hline \multicolumn{6}{|c|}{$\mathrm{Kr}^{3+}\left({ }^{4} \mathrm{~S}^{\circ}\right)+\mathrm{H}\left({ }^{2} \mathrm{~S}\right) \rightarrow \mathrm{Kr}^{2+}+\mathrm{H}^{+}\left({ }^{1} \mathrm{~S}\right)+\Delta E \quad\left({ }^{3} \Sigma^{-},{ }^{5} \Sigma^{-}\right)$} \\
\hline $4 s^{2} 4 p^{43} \mathrm{P}$ & 0.8485 & 2.577 & 0.5047 & ${ }^{3} \Sigma^{-}$ & I \\
\hline $4 s^{2} 4 p^{3} 4 d^{5} D^{\circ}$ & 0.2269 & 8.940 & $1.047 \mathrm{E}-02$ & ${ }^{5} \Sigma^{-}$ & I \\
\hline $4 s^{2} 4 p^{3} 5 s^{5} S^{\circ}$ & 0.1942 & 10.39 & $3.313 \mathrm{E}-03$ & ${ }^{5} \Sigma^{-}$ & I \\
\hline $4 s^{2} 4 p^{3} 4 d^{3} D^{\circ}$ & 0.1816 & 11.10 & $1.870 \mathrm{E}-03$ & ${ }^{3} \Sigma^{-}$ & I \\
\hline $4 s^{2} 4 p^{3} 5 s^{3} s^{\circ}$ & 0.1675 & 12.01 & $8.767 \mathrm{E}-04$ & ${ }^{3} \Sigma^{-}$ & I \\
\hline $4 s^{2} 4 p^{3} 4 d^{3} G^{\circ}$ & 0.1264 & 15.86 & $3.248 \mathrm{E}-05$ & ${ }^{3} \Sigma^{-}$ & I \\
\hline $4 s^{2} 4 p^{3} 5 s^{3} D^{\circ}$ & 0.1099 & 18.23 & $4.025 \mathrm{E}-06$ & ${ }^{3} \Sigma^{-}$ & I \\
\hline \multicolumn{6}{|c|}{$\mathrm{Kr}^{4+}\left({ }^{3} \mathrm{P}\right)+\mathrm{H}\left({ }^{2} \mathrm{~S}\right) \rightarrow \mathrm{Kr}^{3+}+\mathrm{H}^{+}\left({ }^{1} \mathrm{~S}\right)+\Delta E \quad\left({ }^{2} \Sigma^{-},{ }^{2} \Pi,{ }^{4} \Sigma^{-},{ }^{4} \Pi\right)$} \\
\hline $4 \mathrm{~s} 4 \mathrm{p}^{4}{ }^{4} \mathrm{P}$ & 0.8787 & 3.834 & $3.429 \mathrm{E}-02$ & ${ }^{4} \Sigma^{-},{ }^{4} \Pi$ & II \\
\hline $4 s^{2} 4 p^{2} 4 d^{2} P_{a}$ & 0.6847 & 4.775 & 0.1924 & ${ }^{2} \Sigma^{-},{ }^{2} \Pi$ & I \\
\hline $4 s^{2} 4 p^{2} 4 d^{4} F$ & 0.6265 & 5.155 & 0.1533 & ${ }^{4} \Sigma^{-},{ }^{4} \Pi$ & I \\
\hline $4 s^{2} 4 p^{2} 4 d^{4} D$ & 0.6030 & 5.328 & 0.1378 & ${ }^{4} \Pi$ & I \\
\hline $4 s^{2} 4 p^{2} 4 d^{2} F_{a}$ & 0.5937 & 5.400 & 0.1317 & ${ }^{2} \Sigma^{-},{ }^{2} \Pi$ & I \\
\hline $4 \mathrm{~s} 4 \mathrm{p}^{4}{ }^{2} \mathrm{P}$ & 0.5532 & 5.744 & $5.308 \mathrm{E}-03$ & ${ }^{2} \Sigma^{-},{ }^{2} \Pi$ & II \\
\hline $4 s^{2} 4 p^{2} 4 d^{4} P$ & 0.5079 & 6.193 & $7.838 \mathrm{E}-02$ & ${ }^{4} \Sigma^{-},{ }^{4} \Pi$ & I \\
\hline $4 s^{2} 4 p^{2} 5 s{ }^{4} P$ & 0.4901 & 6.394 & $6.834 \mathrm{E}-02$ & ${ }^{4} \Sigma^{-},{ }^{4} \Pi$ & I \\
\hline $4 \mathrm{~s}^{2} 4 \mathrm{p}^{2} 4 \mathrm{~d}^{2} \mathrm{D}_{a}$ & 0.4719 & 6.615 & $5.864 \mathrm{E}-02$ & ${ }^{2} \Pi$ & I \\
\hline $4 s^{2} 4 p^{2} 5 s^{2} P$ & 0.4691 & 6.650 & $5.723 \mathrm{E}-02$ & ${ }^{2} \Sigma^{-},{ }^{2} \Pi$ & I \\
\hline $4 s^{2} 4 p^{2} 4 d^{2} D_{b}$ & 0.4386 & 7.069 & $4.253 \mathrm{E}-02$ & ${ }^{2} \Pi$ & I \\
\hline $4 s^{2} 4 p^{2} 4 d^{2} P_{b}$ & 0.4257 & 7.266 & $3.690 \mathrm{E}-02$ & ${ }^{2} \Sigma^{-},{ }^{2} \Pi$ & I \\
\hline $4 s^{2} 4 p^{2} 4 d^{2} F_{b}$ & 0.4240 & 7.293 & $3.618 \mathrm{E}-02$ & ${ }^{2} \Sigma^{-},{ }^{2} \Pi$ & I \\
\hline $4 s^{2} 4 p^{2} 5 s^{2} D$ & 0.4124 & 7.482 & $3.152 \mathrm{E}-02$ & ${ }^{2} \Pi$ & I \\
\hline $4 s^{2} 4 p^{2} 4 d^{2} D_{c}$ & 0.3710 & 8.259 & $1.766 \mathrm{E}-02$ & ${ }^{2} \Pi$ & I \\
\hline $4 s^{2} 4 p^{2} 5 p^{2} S^{\circ}$ & 0.3595 & 8.508 & $1.461 \mathrm{E}-02$ & ${ }^{2} \Sigma^{-}$ & I \\
\hline $4 s^{2} 4 p^{2} 5 p^{4} D^{\circ}$ & 0.3289 & 9.260 & $8.159 \mathrm{E}-03$ & ${ }^{4} \Sigma^{-},{ }^{4} \Pi$ & I \\
\hline $4 s^{2} 4 p^{2} 5 p^{4} \mathrm{P}^{\circ}$ & 0.3203 & 9.498 & $6.766 \mathrm{E}-03$ & ${ }^{4} \Pi$ & I \\
\hline $4 s^{2} 4 p^{2} 5 p^{4} S^{\circ}$ & 0.3058 & 9.931 & 4.797E-03 & ${ }^{4} \Sigma^{-}$ & I \\
\hline $4 s^{2} 4 p^{2} 5 p^{2} D_{a}^{\circ}$ & 0.3036 & 10.00 & $4.540 \mathrm{E}-03$ & ${ }^{2} \Sigma^{-},{ }^{2} \Pi$ & I \\
\hline $4 s^{2} 4 p^{2} 5 p^{2} \mathrm{P}_{a}^{\circ}$ & 0.2884 & 10.51 & $3.011 \mathrm{E}-03$ & ${ }^{2} \Pi$ & I \\
\hline $4 s^{2} 4 p^{2} 5 p^{2} F^{\circ}$ & 0.2543 & 11.88 & $9.761 \mathrm{E}-04$ & ${ }^{2} \Pi$ & I \\
\hline $4 s^{2} 4 p^{2} 5 p^{2} D_{b}^{\circ}$ & 0.2512 & 12.03 & $8.658 \mathrm{E}-04$ & ${ }^{2} \Sigma^{-},{ }^{2} \Pi$ & I \\
\hline $4 s^{2} 4 p^{2} 5 p^{2} P_{b}^{b}$ & 0.2235 & 13.49 & $2.522 \mathrm{E}-04$ & ${ }^{2} \Pi$ & I \\
\hline
\end{tabular}


N. C. Sterling and P. C. Stancil: Atomic data for neutron-capture elements. III.

Table 1. continued.

\begin{tabular}{|c|c|c|c|c|c|}
\hline $\begin{array}{l}\text { Exit } \\
\text { Channels }^{a}\end{array}$ & $\begin{array}{l}\Delta E \\
\text { (a.u.) }\end{array}$ & $\begin{array}{c}R_{\mathrm{x}} \\
\text { (a.u.) } \\
\end{array}$ & $\begin{array}{c}\Delta U\left(R_{\mathrm{x}}\right) \\
\text { (a.u.) } \\
\end{array}$ & $\begin{array}{c}\text { Molecular } \\
\text { Symmetries }^{b} \\
\end{array}$ & Type \\
\hline \multicolumn{6}{|c|}{$\mathrm{Kr}^{5+}\left({ }^{2} \mathrm{P}^{\circ}\right)+\mathrm{H}\left({ }^{2} \mathrm{~S}\right) \rightarrow \mathrm{Kr}^{4+}+\mathrm{H}^{+}\left({ }^{1} \mathrm{~S}\right)+\Delta E \quad\left({ }^{1} \Sigma^{+},{ }^{1} \Pi,{ }^{3} \Sigma^{+},{ }^{3} \Pi\right)$} \\
\hline $4 s^{2} 4 p 4 d^{3} P^{\circ}$ & 0.9083 & 4.841 & 0.1851 & ${ }^{3} \Sigma^{+},{ }^{3} \Pi$ & I \\
\hline $4 s^{2} 4 p 4 d^{3} D^{\circ}$ & 0.8781 & 4.998 & 0.1687 & ${ }^{3} \Pi$ & I \\
\hline $4 s^{2} 4 p 4 d^{1} F^{\circ}$ & 0.8110 & 5.344 & 0.1364 & ${ }^{1} \Sigma^{+},{ }^{1} \Pi$ & I \\
\hline $4 s^{2} 4 p 4 d{ }^{1} P^{\circ}$ & 0.7946 & 5.437 & 0.1287 & ${ }^{1} \Sigma^{+},{ }^{1} \Pi$ & I \\
\hline $4 s^{2} 4 p 5 s^{3} \mathrm{P}^{\circ}$ & 0.7633 & 5.619 & 0.1146 & ${ }^{3} \Sigma^{+},{ }^{3} \Pi$ & I \\
\hline $4 s^{2} 4 p 5 s^{1} P^{\circ}$ & 0.7342 & 5.816 & 0.1008 & ${ }^{1} \Sigma^{+},{ }^{1} \Pi$ & I \\
\hline $4 s^{2} 4 p 5 p^{1} P$ & 0.5858 & 7.094 & $4.177 \mathrm{E}-02$ & ${ }^{1} \Pi$ & I \\
\hline $4 s^{2} 4 p 5 p^{3} D$ & 0.5761 & 7.202 & $3.865 \mathrm{E}-02$ & ${ }^{3} \Sigma^{+},{ }^{3} \Pi$ & I \\
\hline $4 s^{2} 4 p 5 p^{3} P$ & 0.5554 & 7.446 & $3.237 \mathrm{E}-02$ & ${ }^{3} \Pi$ & I \\
\hline $4 s^{2} 4 p 5 p^{3} s$ & 0.5395 & 7.646 & $2.794 \mathrm{E}-02$ & ${ }^{3} \Sigma^{+}$ & I \\
\hline $4 s^{2} 4 p 5 p^{1} D$ & 0.5264 & 7.822 & $2.452 \mathrm{E}-02$ & ${ }^{1} \Sigma^{+},{ }^{1} \Pi$ & I \\
\hline $4 s^{2} 4 p 5 p^{1} S$ & 0.4759 & 8.592 & $1.370 \mathrm{E}-02$ & ${ }^{1} \Sigma^{+}$ & $\mathrm{I}$ \\
\hline \multicolumn{6}{|c|}{$\mathrm{Rb}^{0}\left({ }^{2} \mathrm{~S}\right)+\mathrm{H}^{+}\left({ }^{1} \mathrm{~S}\right) \rightleftharpoons \mathrm{Rb}^{+}+\mathrm{H}\left({ }^{2} \mathrm{~S}\right)+\Delta E \quad\left({ }^{2} \Sigma^{+}\right)$} \\
\hline $4 s^{2} 4 p^{6}{ }^{1} s$ & 0.3462 & 10.46 & 0.3332 & ${ }^{2} \Sigma^{+}$ & $\mathrm{I}$ \\
\hline \multicolumn{6}{|c|}{$\mathrm{Rb}^{2+}\left({ }^{2} \mathrm{P}^{\circ}\right)+\mathrm{H}\left({ }^{2} \mathrm{~S}\right) \rightarrow \mathrm{Rb}^{+}+\mathrm{H}^{+}\left({ }^{1} \mathrm{~S}\right)+\Delta E \quad\left({ }^{1} \Sigma^{+},{ }^{1} \Pi,{ }^{3} \Sigma^{+},{ }^{3} \Pi\right)$} \\
\hline $4 s^{2} 4 p^{6}{ }^{1} S$ & 0.5031 & 2.175 & 0.5374 & ${ }^{1} \Sigma^{+}$ & I \\
\hline \multicolumn{6}{|c|}{$\mathrm{Rb}^{3+}\left({ }^{3} \mathrm{P}\right)+\mathrm{H}\left({ }^{2} \mathrm{~S}\right) \rightarrow \mathrm{Rb}^{2+}+\mathrm{H}^{+}\left({ }^{1} \mathrm{~S}\right)+\Delta E \quad\left({ }^{2} \Sigma^{-},{ }^{2} \Pi,{ }^{4} \Sigma^{-},{ }^{4} \Pi\right)$} \\
\hline $4 s^{2} 4 p^{4} 4 d^{4} D$ & 0.2275 & 8.917 & $1.066 \mathrm{E}-02$ & ${ }^{4} \Pi$ & I \\
\hline $4 s^{2} 4 p^{4} 4 d^{4} F$ & 0.1798 & 11.20 & $1.710 \mathrm{E}-03$ & ${ }^{4} \Sigma^{-},{ }^{4} \Pi$ & I \\
\hline $4 s^{2} 4 p^{4} 5 s^{2} D$ & 0.1629 & 12.34 & $6.637 \mathrm{E}-04$ & ${ }^{2} \Pi$ & I \\
\hline $4 s^{2} 4 p^{4} 4 d^{2} P$ & 0.1593 & 12.62 & $5.269 \mathrm{E}-04$ & ${ }^{2} \Sigma^{-},{ }^{2} \Pi$ & I \\
\hline $4 s^{2} 4 p^{4} 4 d^{4} P$ & 0.1559 & 12.89 & 4.192E-04 & ${ }^{4} \Sigma^{-},{ }^{4} \Pi$ & I \\
\hline $4 s^{2} 4 p^{4} 4 d^{2} D$ & 0.1463 & 13.73 & $2.062 \mathrm{E}-04$ & ${ }^{2} \Pi$ & I \\
\hline $4 s^{2} 4 p^{4} 4 d^{2} F$ & 0.1428 & 14.06 & $1.552 \mathrm{E}-04$ & ${ }^{2} \Sigma^{-},{ }^{2} \Pi$ & I \\
\hline $4 s^{2} 4 p^{4} 5 s^{2} P$ & 0.1261 & 15.90 & $3.142 \mathrm{E}-05$ & ${ }^{2} \Sigma^{-},{ }^{2} \Pi$ & I \\
\hline $4 s^{2} 4 p^{4} 4 d^{2} D$ & 0.1219 & 16.45 & $1.950 \mathrm{E}-05$ & ${ }^{2} \Pi$ & I \\
\hline \multicolumn{6}{|c|}{$\mathrm{Rb}^{4+}\left({ }^{4} \mathrm{~S}^{\circ}\right)+\mathrm{H}\left({ }^{2} \mathrm{~S}\right) \rightarrow \mathrm{Rb}^{3+}+\mathrm{H}^{+}\left({ }^{1} \mathrm{~S}\right)+\Delta E \quad\left({ }^{3} \Sigma^{-},{ }^{5} \Sigma^{-}\right)$} \\
\hline $4 s^{2} 4 p^{3} 4 d^{5} D^{\circ}$ & 0.6392 & 5.043 & 0.1642 & $5 \Sigma^{-}$ & I \\
\hline $4 s^{2} 4 p^{3} 4 d^{3} D_{a}^{\circ}$ & 0.5865 & 5.458 & 0.1270 & ${ }^{3} \Sigma^{-}$ & I \\
\hline $4 s^{2} 4 p^{3} 4 d^{3} F^{\circ}$ & 0.5553 & 5.724 & 0.1070 & ${ }^{3} \Sigma^{-}$ & I \\
\hline $4 s^{2} 4 p^{3} 4 d^{3} G^{\circ}$ & 0.5237 & 6.027 & $8.764 \mathrm{E}-02$ & ${ }^{3} \Sigma^{-}$ & I \\
\hline $4 s^{2} 4 p^{3} 5 s^{5} S^{\circ}$ & 0.4923 & 6.364 & $6.976 \mathrm{E}-02$ & ${ }^{5} \Sigma^{-}$ & I \\
\hline $4 s^{2} 4 p^{3} 5 s^{3} S^{\circ}$ & 0.4619 & 6.744 & $5.357 \mathrm{E}-02$ & ${ }^{3} \Sigma^{-}$ & I \\
\hline $4 s^{2} 4 p^{3} 4 d^{3} D_{b}^{\circ}$ & 0.4225 & 7.316 & $3.558 \mathrm{E}-02$ & ${ }^{3} \Sigma^{-}$ & I \\
\hline $4 s^{2} 4 p^{3} 5 s^{3} D^{b}$ & 0.3955 & 7.778 & $2.534 \mathrm{E}-02$ & ${ }^{3} \Sigma^{-}$ & I \\
\hline $4 s^{2} 4 p^{3} 4 d^{3} S^{\circ}$ & 0.3935 & 7.815 & $2.465 \mathrm{E}-02$ & ${ }^{3} \Sigma^{-}$ & I \\
\hline $4 s^{2} 4 p^{3} 4 d^{3} D_{c}^{\circ}$ & 0.3931 & 7.822 & $2.452 \mathrm{E}-02$ & ${ }^{3} \Sigma^{-}$ & I \\
\hline $4 s^{2} 4 p^{3} 5 p^{5} P^{2}$ & 0.3123 & 9.732 & $5.622 \mathrm{E}-03$ & ${ }^{5} \Sigma^{-}$ & I \\
\hline $4 s^{2} 4 p^{3} 5 p^{3} P_{a}$ & 0.2901 & 10.45 & $3.161 \mathrm{E}-03$ & ${ }^{3} \Sigma^{-}$ & I \\
\hline $4 s^{2} 4 p^{3} 5 p^{3} F$ & 0.2189 & 13.77 & $1.988 \mathrm{E}-04$ & ${ }^{3} \Sigma^{-}$ & I \\
\hline $4 s^{2} 4 p^{3} 5 p^{3} P_{b}$ & 0.1942 & 15.50 & 4.465E-05 & ${ }^{3} \Sigma^{-}$ & I \\
\hline \multicolumn{6}{|c|}{$\mathrm{Rb}^{5+}\left({ }^{3} \mathrm{P}\right)+\mathrm{H}\left({ }^{2} \mathrm{~S}\right) \rightarrow \mathrm{Rb}^{4+}+\mathrm{H}^{+}\left({ }^{1} \mathrm{~S}\right)+\Delta E \quad\left({ }^{2} \Sigma^{-},{ }^{2} \Pi,{ }^{4} \Sigma^{-},{ }^{4} \Pi\right)$} \\
\hline $4 s 4 p^{4}{ }^{4} \mathrm{P}$ & 1.3832 & 3.388 & 4.999E-02 & ${ }^{4} \Sigma^{-},{ }^{4} \Pi$ & II \\
\hline $4 \mathrm{~s} 4 \mathrm{p}^{4}{ }^{2} \mathrm{D}$ & 1.2509 & 3.706 & $3.833 \mathrm{E}-02$ & ${ }^{2} \Pi$ & II \\
\hline $4 s 4 p^{4}{ }^{2} \mathrm{P}$ & 1.1465 & 3.996 & $2.969 \mathrm{E}-02$ & ${ }^{2} \Sigma^{-},{ }^{2} \Pi$ & II \\
\hline $4 s^{2} 4 p^{2} 5 s^{4} \mathrm{P}$ & 0.7897 & 5.465 & 0.1264 & ${ }^{4} \Sigma^{-},{ }^{4} \Pi$ & I \\
\hline $4 s^{2} 4 p^{2} 5 s^{2} P$ & 0.7817 & 5.513 & 0.1226 & ${ }^{2} \Sigma^{-},{ }^{2} \Pi$ & I \\
\hline $4 s^{2} 4 p^{2} 5 s^{2} D$ & 0.7110 & 5.979 & 9.049E-02 & ${ }^{2} \Pi$ & $\mathrm{I}$ \\
\hline \multicolumn{6}{|c|}{$\mathrm{Xe}^{0}\left({ }^{1} \mathrm{~S}\right)+\mathrm{H}^{+}\left({ }^{1} \mathrm{~S}\right) \rightleftharpoons \mathrm{Xe}^{+}+\mathrm{H}\left({ }^{2} \mathrm{~S}\right)+\Delta E \quad\left({ }^{1} \Sigma^{+}\right)$} \\
\hline $5 s^{2} 5 p^{5}{ }^{2} \mathrm{P}^{\circ}$ & 0.0380 & 7.933 & $3.515 \mathrm{E}-02$ & ${ }^{1} \Sigma^{+}$ & $\mathrm{I}$ \\
\hline \multicolumn{6}{|c|}{$\mathrm{Xe}^{2+}\left({ }^{3} \mathrm{P}\right)+\mathrm{H}\left({ }^{2} \mathrm{~S}\right) \rightarrow \mathrm{Xe}^{+}+\mathrm{H}^{+}\left({ }^{1} \mathrm{~S}\right)+\Delta E \quad\left({ }^{2} \Sigma^{-},{ }^{2} \Pi,{ }^{4} \Sigma^{-},{ }^{4} \Pi\right)$} \\
\hline $5 \mathrm{~s}^{2} 5 \mathrm{p}^{5}{ }^{2} \mathrm{P}^{\circ}$ & 0.2551 & 4.078 & 0.2817 & ${ }^{2} \Sigma^{-},{ }^{2} \Pi$ & I \\
\hline \multicolumn{6}{|c|}{$\mathrm{Xe}^{3+}\left({ }^{4} S^{\circ}\right)+\mathrm{H}\left({ }^{2} \mathrm{~S}\right) \rightarrow \mathrm{Xe}^{2+}+\mathrm{H}^{+}\left({ }^{1} \mathrm{~S}\right)+\Delta E$} \\
\hline $5 s^{2} 5 p^{3} 5 d^{5} D^{\circ}$ & 0.1307 & 15.35 & 5.097E-05 & ${ }^{5} \Sigma^{-}$ & $\mathrm{I}$ \\
\hline
\end{tabular}


Table 1. continued.

\begin{tabular}{|c|c|c|c|c|c|}
\hline $\begin{array}{l}\text { Exit } \\
\text { Channels }^{a}\end{array}$ & $\begin{array}{l}\Delta E \\
\text { (a.u.) }\end{array}$ & $\begin{array}{c}R_{\mathrm{x}} \\
\text { (a.u.) }\end{array}$ & $\begin{array}{c}\Delta U\left(R_{\mathrm{x}}\right) \\
\text { (a.u.) }\end{array}$ & $\begin{array}{c}\text { Molecular } \\
\text { Symmetries }^{b}\end{array}$ & Type \\
\hline \multicolumn{4}{|c|}{$\mathrm{Xe}^{4+}\left({ }^{3} \mathrm{P}\right)+\mathrm{H}\left({ }^{2} \mathrm{~S}\right) \rightarrow \mathrm{Xe}^{3+}+\mathrm{H}^{+}\left({ }^{1} \mathrm{~S}\right)+\Delta E$} & \multicolumn{2}{|c|}{$\left({ }^{2} \Sigma^{-},{ }^{2} \Pi,{ }^{4} \Sigma^{-},{ }^{4} \Pi\right)$} \\
\hline $5 s 5 p^{4}{ }^{4} \mathrm{P}$ & 0.5315 & 5.947 & $4.283 \mathrm{E}-03$ & ${ }^{4} \Sigma^{-},{ }^{4} \Pi$ & II \\
\hline $5 s 5 p^{4}{ }^{2} \mathrm{D}$ & 0.4386 & 7.068 & $1.259 \mathrm{E}-03$ & ${ }^{2} \Pi$ & II \\
\hline $5 \mathrm{~s}^{2} 5 \mathrm{p}^{2} 5 \mathrm{~d}^{2} \mathrm{P}_{a}$ & 0.3920 & 7.843 & $2.414 \mathrm{E}-02$ & ${ }^{2} \Sigma^{-},{ }^{2} \Pi$ & I \\
\hline $5 s^{2} 5 p^{2} 5 d^{4} F$ & 0.3608 & 8.479 & $1.494 \mathrm{E}-02$ & ${ }^{4} \Sigma^{-},{ }^{4} \Pi$ & I \\
\hline $5 s^{2} 5 p^{2} 5 d^{2} F_{a}$ & 0.3494 & 8.741 & $1.222 \mathrm{E}-02$ & ${ }^{2} \Sigma^{-},{ }^{2} \Pi$ & I \\
\hline $5 s^{2} 5 p^{2} 5 d^{4} D$ & 0.3172 & 9.587 & $6.306 \mathrm{E}-03$ & ${ }^{4} \Pi$ & I \\
\hline $5 \mathrm{~s}^{2} 5 \mathrm{p}^{2} 5 \mathrm{~d}^{4} \mathrm{P}$ & 0.2713 & 11.15 & $1.781 \mathrm{E}-03$ & ${ }^{4} \Sigma^{-},{ }^{4} \Pi$ & I \\
\hline $5 s^{2} 5 p^{2} 5 d^{2} G$ & 0.2647 & 11.43 & $1.425 \mathrm{E}-03$ & ${ }^{2} \Pi$ & I \\
\hline $5 s^{2} 5 p^{2} 6 s{ }^{4} P$ & 0.2450 & 12.32 & $6.749 \mathrm{E}-04$ & ${ }^{4} \Sigma^{-},{ }^{4} \Pi$ & I \\
\hline $5 \mathrm{~s}^{2} 5 \mathrm{p}^{2} 5 \mathrm{~d}^{2} \mathrm{D}_{a}$ & 0.2437 & 12.39 & $6.397 \mathrm{E}-04$ & ${ }^{2} \Pi$ & I \\
\hline $5 s^{2} 5 p^{2} 6 s^{2} P$ & 0.2237 & 13.48 & $2.548 \mathrm{E}-04$ & ${ }^{2} \Sigma^{-},{ }^{2} \Pi$ & I \\
\hline $5 s^{2} 5 p^{2} 5 d^{2} F_{b}$ & 0.1969 & 15.29 & $5.361 \mathrm{E}-05$ & ${ }^{2} \Sigma^{-},{ }^{2} \Pi$ & I \\
\hline $5 \mathrm{~s}^{2} 5 \mathrm{p}^{2} 5 \mathrm{~d}^{2} \mathrm{D}_{b}$ & 0.1935 & 15.55 & $4.253 \mathrm{E}-05$ & ${ }^{2} \Pi$ & I \\
\hline $5 \mathrm{~s}^{2} 5 \mathrm{p}^{2} 5 \mathrm{~d}^{2} \mathrm{P}_{b}$ & 0.1867 & 16.12 & $2.605 \mathrm{E}-05$ & ${ }^{2} \Sigma^{-},{ }^{2} \Pi$ & I \\
\hline $5 s^{2} 5 p^{2} 4 f^{4} G^{\circ}$ & 0.1645 & 18.27 & $3.870 \mathrm{E}-06$ & ${ }^{4} \Sigma^{-},{ }^{4} \Pi$ & I \\
\hline \multicolumn{6}{|c|}{$\mathrm{Xe}^{5+}\left({ }^{2} \mathrm{P}^{\circ}\right)+\mathrm{H}\left({ }^{2} \mathrm{~S}\right) \rightarrow \mathrm{Xe}^{4+}+\mathrm{H}^{+}\left({ }^{1} \mathrm{~S}\right)+\Delta E \quad\left({ }^{1} \Sigma^{+},{ }^{1} \Pi,{ }^{3} \Sigma^{+},{ }^{3} \Pi\right)$} \\
\hline $5 s^{2} 5 p 4 f^{3} D$ & 0.9346 & 4.745 & 0.1958 & ${ }^{3} \Sigma^{+},{ }^{3} \Pi$ & I \\
\hline $5 s^{2} 5 p 4 f^{1} D$ & 0.8902 & 4.942 & 0.1744 & ${ }^{1} \Sigma^{+},{ }^{1} \Pi$ & I \\
\hline $5 s^{2} 5 p 6 p^{3} D$ & 0.7893 & 5.468 & 0.1262 & ${ }^{3} \Sigma^{+},{ }^{3} \Pi$ & I \\
\hline $5 \mathrm{~s}^{2} 5 \mathrm{p} 6 \mathrm{p}^{3} \mathrm{P}$ & 0.7835 & 5.502 & 0.1235 & ${ }^{3} \Pi$ & I \\
\hline $5 \mathrm{~s}^{2} 5 \mathrm{p} 6 \mathrm{p}^{1} \mathrm{P}$ & 0.7696 & 5.586 & 0.1170 & ${ }^{1} \Pi$ & I \\
\hline $5 s^{2} 5 p 6 p^{3} S$ & 0.7487 & 5.719 & 0.1074 & ${ }^{3} \Sigma^{+}$ & I \\
\hline $5 s^{2} 5 p 6 p^{1} D$ & 0.7361 & 5.803 & 0.1017 & ${ }^{1} \Sigma^{+},{ }^{1} \Pi$ & I \\
\hline $5 s^{2} 5 p 6 p^{1} S$ & 0.6947 & 6.100 & $8.346 \mathrm{E}-02$ & ${ }^{1} \Sigma^{+}$ & I \\
\hline
\end{tabular}

Notes. The table includes exit channels, molecular symmetries, energy defects $\Delta E$, avoided crossing or interaction distances $R_{\mathrm{x}}$, and the energy separations of the adiabatic potentials $\Delta U\left(R_{\mathrm{x}}\right)$. The molecular symmetries of each entrance channel is given in parentheses after the reaction. (a) Exit channels refer to states in the resulting ion after $\mathrm{CT}$ with $\mathrm{H}$ or $\mathrm{H}^{+}$. Only information about electrons in the valence principal quantum number shell is given. Some terms are given a lettered sub-index (e.g., $\left.{ }^{2} \mathrm{D}_{a}\right)$ if multiple terms with the same configuration and orbital and spin angular momenta are exoergic exit channels. Note that exit channels with rate coefficients smaller than $10^{-14} \mathrm{~cm}^{3} \mathrm{~s}^{-1}$ (the canonical radiative CT rate) in the temperature range $10^{2}-10^{6} \mathrm{~K}$ are not listed, unless no exoergic exit channels with larger rate coefficients exist. ${ }^{(b)}$ Only molecular symmetries that couple to the entrance channel are listed.

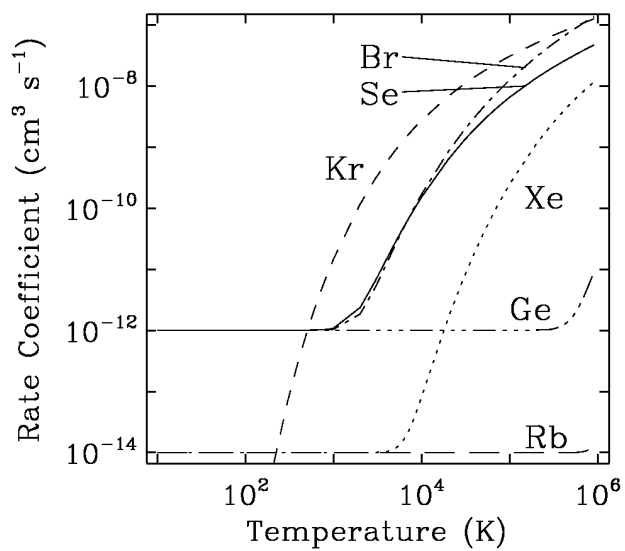

Fig. 4. Total rate coefficients for $\mathrm{CT}$ ionization of the indicated neutral species.

corresponding to the molecular symmetries involved in the recombination and ionization reactions, $\Delta E_{\infty}$ is the asymptotic energy defect, and $k_{\mathrm{B}}$ the Boltzmann constant. We note that Eq. (15) was applied only to state-resolved rate coefficients. In the case of $\mathrm{Kr}$, for which CT recombination of singly-charged ions is exothermic, the CT ionization rate coefficients were computed in an analogous manner. For neutral Kr, the energy defect is very small (corresponding to $4700 \mathrm{~K}$ ), and hence the CT ionization reaction can operate efficiently in photoionized nebulae. CT recombination of singly-charged ions is less important at photoionized temperatures for the other elements (see Fig. 3), as the lowest energy defect is that of Xe (corresponding to $17000 \mathrm{~K}$ ).

State-resolved rate coefficients are important for understanding the emission line spectra of photoionized nebulae, since CT populates excited states from which emission lines can be measured (e.g., Kingdon \& Ferland 1996). We therefore provide final-state resolved rate coefficients for $\mathrm{CT}$ recombination in Table 4 and for CT ionization in Table 5 (both available at the CDS).

The derived abundances of Se and $\mathrm{Kr}$ have explicitly been shown to be sensitive to uncertainties in $\mathrm{CT}$ rate coefficients (Sterling et al. 2007), and other trans-iron elements are expected to be similarly sensitive. Unfortunately, it is not possible to ascertain robust uncertainties for CT rate coefficients computed with the LZ or Demkov approximations without accompanying QMOCC calculations. Butler \& Dalgarno (1980b) found that their LZ rate coefficients agreed with quantal calculations to within a factor of three for ions of light elements with large CT rate coefficients, but that the disagreement could exceed an order of magnitude in the case of systems with small rate coefficients. No such comparison is possible for the heavy ions we have investigated, since QMOCC computations are not available for any 
N. C. Sterling and P. C. Stancil: Atomic data for neutron-capture elements. III.

Table 2. Total charge transfer recombination rate coefficients $\left(\mathrm{cm}^{3} \mathrm{~s}^{-1}\right)$ for the reactions $\mathrm{X}^{q+}+\mathrm{H} \rightarrow \mathrm{X}^{(q-1)+}+\mathrm{H}^{+}+\delta E$.

\begin{tabular}{|c|c|c|c|c|c|c|c|c|c|c|}
\hline \multirow{3}{*}{$\begin{array}{l}\text { Projectile } \\
\text { Ion } \mathrm{X}^{q^{+}}\end{array}$} & \multicolumn{10}{|c|}{$T(\mathrm{~K})$} \\
\hline & $1.0 \mathrm{E}+02$ & $2.0 \mathrm{E}+02$ & $4.0 \mathrm{E}+02$ & $6.0 \mathrm{E}+02$ & $8.0 \mathrm{E}+02$ & $1.0 \mathrm{E}+03$ & $2.0 \mathrm{E}+03$ & $4.0 \mathrm{E}+03$ & $6.0 \mathrm{E}+03$ & $8.0 \mathrm{E}+03$ \\
\hline & $1.0 \mathrm{E}+04$ & $2.0 \mathrm{E}+04$ & $4.0 \mathrm{E}+04$ & $6.0 \mathrm{E}+04$ & $8.0 \mathrm{E}+04$ & $1.0 \mathrm{E}+05$ & $2.0 \mathrm{E}+05$ & $4.0 \mathrm{E}+05$ & $6.0 \mathrm{E}+05$ & $8.0 \mathrm{E}+05$ \\
\hline \multirow[t]{2}{*}{$\mathrm{Ge}^{+}$} & $0.00 \mathrm{E}+00$ & $0.00 \mathrm{E}+00$ & $7.94 \mathrm{E}-83$ & $1.68 \mathrm{E}-59$ & $7.72 \mathrm{E}-48$ & $7.67 \mathrm{E}-41$ & $7.59 \mathrm{E}-27$ & $7.54 \mathrm{E}-20$ & $1.62 \mathrm{E}-17$ & $2.38 \mathrm{E}-16$ \\
\hline & $1.19 \mathrm{E}-15$ & $2.99 \mathrm{E}-14$ & $1.50 \mathrm{E}-13$ & $2.56 \mathrm{E}-13$ & $3.35 \mathrm{E}-13$ & $3.94 \mathrm{E}-13$ & $5.45 \mathrm{E}-13$ & 7.47E-13 & $1.56 \mathrm{E}-12$ & $4.03 \mathrm{E}-12$ \\
\hline \multirow[t]{2}{*}{$\mathrm{Ge}^{2+}$} & 7.67E-13 & $5.37 \mathrm{E}-12$ & $3.12 \mathrm{E}-11$ & $7.81 \mathrm{E}-11$ & $1.42 \mathrm{E}-10$ & $2.20 \mathrm{E}-10$ & 7.32E-10 & $1.96 \mathrm{E}-09$ & $3.21 \mathrm{E}-09$ & 4.39E-09 \\
\hline & $5.50 \mathrm{E}-09$ & $1.01 \mathrm{E}-08$ & $1.64 \mathrm{E}-08$ & $2.08 \mathrm{E}-08$ & $2.41 \mathrm{E}-08$ & $2.69 \mathrm{E}-08$ & $3.58 \mathrm{E}-08$ & $4.50 \mathrm{E}-08$ & $5.02 \mathrm{E}-08$ & $5.38 \mathrm{E}-08$ \\
\hline \multirow[t]{2}{*}{$\mathrm{Ge}^{3+}$} & $1.00 \mathrm{E}-14$ & $1.00 \mathrm{E}-14$ & $1.00 \mathrm{E}-14$ & $1.00 \mathrm{E}-14$ & $1.00 \mathrm{E}-14$ & $1.00 \mathrm{E}-14$ & $1.00 \mathrm{E}-14$ & $1.00 \mathrm{E}-14$ & $1.00 \mathrm{E}-14$ & $1.00 \mathrm{E}-14$ \\
\hline & $1.00 \mathrm{E}-14$ & $3.36 \mathrm{E}-14$ & $6.03 \mathrm{E}-13$ & $2.54 \mathrm{E}-12$ & $6.37 \mathrm{E}-12$ & $1.23 \mathrm{E}-11$ & $7.46 \mathrm{E}-11$ & $3.23 \mathrm{E}-10$ & $6.66 \mathrm{E}-10$ & $1.06 \mathrm{E}-09$ \\
\hline \multirow[t]{2}{*}{$\mathrm{Ge}^{4+}$} & 4.50E-09 & $4.56 \mathrm{E}-09$ & 4.56E-09 & 4.57E-09 & 4.60E-09 & 4.64E-09 & $4.90 \mathrm{E}-09$ & $5.38 \mathrm{E}-09$ & $5.76 \mathrm{E}-09$ & $6.05 \mathrm{E}-09$ \\
\hline & 6.30E-09 & 7.11E-09 & 7.93E-09 & 8.40E-09 & 8.72E-09 & 8.95E-09 & 9.63E-09 & $1.02 \mathrm{E}-08$ & $1.05 \mathrm{E}-08$ & $1.07 \mathrm{E}-08$ \\
\hline \multirow[t]{2}{*}{$\mathrm{Ge}^{5+}$} & 6.34E-09 & $6.40 \mathrm{E}-09$ & 6.37E-09 & 6.33E-09 & $6.31 \mathrm{E}-09$ & 6.32E-09 & $6.52 \mathrm{E}-09$ & 7.10E-09 & $7.66 \mathrm{E}-09$ & $8.15 \mathrm{E}-09$ \\
\hline & 8.58E-09 & $1.02 \mathrm{E}-08$ & $1.24 \mathrm{E}-08$ & $1.41 \mathrm{E}-08$ & $1.55 \mathrm{E}-08$ & $1.67 \mathrm{E}-08$ & 2.19E-08 & $2.98 \mathrm{E}-08$ & $3.61 \mathrm{E}-08$ & $4.15 \mathrm{E}-08$ \\
\hline \multirow[t]{2}{*}{$\mathrm{Se}^{+}$} & $0.00 \mathrm{E}+00$ & $0.00 \mathrm{E}+00$ & $0.00 \mathrm{E}+00$ & $5.88 \mathrm{E}-83$ & $1.24 \mathrm{E}-71$ & $2.96 \mathrm{E}-64$ & $4.67 \mathrm{E}-47$ & $8.96 \mathrm{E}-36$ & $5.41 \mathrm{E}-31$ & $3.36 \mathrm{E}-28$ \\
\hline & $2.58 \mathrm{E}-26$ & $1.12 \mathrm{E}-21$ & $2.28 \mathrm{E}-18$ & 7.39E-17 & $6.27 \mathrm{E}-16$ & $2.80 \mathrm{E}-15$ & $1.43 \mathrm{E}-13$ & $3.09 \mathrm{E}-12$ & $1.38 \mathrm{E}-11$ & $3.55 \mathrm{E}-11$ \\
\hline \multirow[t]{2}{*}{$\mathrm{Se}^{2+}$} & $1.00 \mathrm{E}-14$ & $1.00 \mathrm{E}-14$ & $1.00 \mathrm{E}-14$ & $1.00 \mathrm{E}-14$ & $1.00 \mathrm{E}-14$ & $1.00 \mathrm{E}-14$ & $1.00 \mathrm{E}-14$ & $1.00 \mathrm{E}-14$ & $1.00 \mathrm{E}-14$ & $1.00 \mathrm{E}-14$ \\
\hline & $1.00 \mathrm{E}-14$ & $1.00 \mathrm{E}-14$ & $1.00 \mathrm{E}-14$ & $1.00 \mathrm{E}-14$ & $1.00 \mathrm{E}-14$ & $1.55 \mathrm{E}-14$ & $4.00 \mathrm{E}-13$ & $5.45 \mathrm{E}-12$ & $1.98 \mathrm{E}-11$ & $4.50 \mathrm{E}-11$ \\
\hline \multirow[t]{2}{*}{$\mathrm{Se}^{3+}$} & $6.92 \mathrm{E}-13$ & 7.29E-13 & $7.31 \mathrm{E}-13$ & $7.14 \mathrm{E}-13$ & $6.98 \mathrm{E}-13$ & $6.85 \mathrm{E}-13$ & $6.55 \mathrm{E}-13$ & $6.34 \mathrm{E}-13$ & $6.19 \mathrm{E}-13$ & $6.10 \mathrm{E}-13$ \\
\hline & $6.05 \mathrm{E}-13$ & $5.96 \mathrm{E}-13$ & $5.92 \mathrm{E}-13$ & $5.90 \mathrm{E}-13$ & $5.90 \mathrm{E}-13$ & $5.90 \mathrm{E}-13$ & $5.91 \mathrm{E}-13$ & $5.93 \mathrm{E}-13$ & $6.06 \mathrm{E}-13$ & $6.51 \mathrm{E}-13$ \\
\hline \multirow[t]{2}{*}{$\mathrm{Se}^{4+}$} & $6.15 \mathrm{E}-10$ & $5.96 \mathrm{E}-10$ & $5.98 \mathrm{E}-10$ & $6.29 \mathrm{E}-10$ & $6.72 \mathrm{E}-10$ & 7.23E-10 & $1.03 \mathrm{E}-09$ & $1.68 \mathrm{E}-09$ & 2.31E-09 & 2.91E-09 \\
\hline & $3.48 \mathrm{E}-09$ & $5.96 \mathrm{E}-09$ & 9.77E-09 & $1.27 \mathrm{E}-08$ & $1.52 \mathrm{E}-08$ & $1.72 \mathrm{E}-08$ & $2.47 \mathrm{E}-08$ & 3.35E-08 & $3.90 \mathrm{E}-08$ & 4.30E-08 \\
\hline \multirow[t]{2}{*}{$\mathrm{Se}^{5+}$} & $3.70 \mathrm{E}-10$ & $3.90 \mathrm{E}-10$ & $4.00 \mathrm{E}-10$ & $3.98 \mathrm{E}-10$ & $3.94 \mathrm{E}-10$ & $3.89 \mathrm{E}-10$ & $3.73 \mathrm{E}-10$ & $3.59 \mathrm{E}-10$ & $3.54 \mathrm{E}-10$ & $3.53 \mathrm{E}-10$ \\
\hline & $3.53 \mathrm{E}-10$ & $3.61 \mathrm{E}-10$ & 4.04E-10 & $4.79 \mathrm{E}-10$ & $5.82 \mathrm{E}-10$ & 7.09E-10 & 1.56E-09 & 3.66E-09 & $5.87 \mathrm{E}-09$ & $8.03 \mathrm{E}-09$ \\
\hline $\mathrm{Br}^{+}$ & $1.68 \mathrm{E}-93$ & $4.10 \mathrm{E}-53$ & $6.40 \mathrm{E}-33$ & $3.45 \mathrm{E}-26$ & $8.00 \mathrm{E}-23$ & $8.37 \mathrm{E}-21$ & $9.15 \mathrm{E}-17$ & $9.56 \mathrm{E}-15$ & $4.51 \mathrm{E}-14$ & $9.78 \mathrm{E}-14$ \\
\hline & $1.56 \mathrm{E}-13$ & $3.95 \mathrm{E}-13$ & $6.73 \mathrm{E}-13$ & $1.04 \mathrm{E}-12$ & $1.81 \mathrm{E}-12$ & $3.20 \mathrm{E}-12$ & $2.36 \mathrm{E}-11$ & $1.37 \mathrm{E}-10$ & $3.27 \mathrm{E}-10$ & $5.67 \mathrm{E}-10$ \\
\hline $\mathrm{Br}^{2+}$ & $1.00 \mathrm{E}-14$ & $1.00 \mathrm{E}-14$ & $1.00 \mathrm{E}-14$ & $1.00 \mathrm{E}-14$ & $1.00 \mathrm{E}-14$ & $1.00 \mathrm{E}-14$ & $1.00 \mathrm{E}-14$ & $1.00 \mathrm{E}-14$ & $1.00 \mathrm{E}-14$ & $1.00 \mathrm{E}-14$ \\
\hline & $1.00 \mathrm{E}-14$ & $1.00 \mathrm{E}-14$ & $1.00 \mathrm{E}-14$ & $1.00 \mathrm{E}-14$ & $1.00 \mathrm{E}-14$ & $1.00 \mathrm{E}-14$ & $1.00 \mathrm{E}-14$ & $1.00 \mathrm{E}-14$ & $1.00 \mathrm{E}-14$ & $1.00 \mathrm{E}-14$ \\
\hline $\mathrm{Br}^{3+}$ & $5.30 \mathrm{E}-10$ & $5.44 \mathrm{E}-10$ & $5.52 \mathrm{E}-10$ & $5.54 \mathrm{E}-10$ & $5.55 \mathrm{E}-10$ & $5.55 \mathrm{E}-10$ & $5.50 \mathrm{E}-10$ & $5.37 \mathrm{E}-10$ & $5.29 \mathrm{E}-10$ & $5.25 \mathrm{E}-10$ \\
\hline & $5.23 \mathrm{E}-10$ & $5.26 \mathrm{E}-10$ & $5.41 \mathrm{E}-10$ & $5.55 \mathrm{E}-10$ & $5.66 \mathrm{E}-10$ & $5.75 \mathrm{E}-10$ & $6.04 \mathrm{E}-10$ & $6.32 \mathrm{E}-10$ & $6.48 \mathrm{E}-10$ & $6.58 \mathrm{E}-10$ \\
\hline $\mathrm{Br}^{4+}$ & 3.89E-09 & $3.88 \mathrm{E}-09$ & 3.86E-09 & $3.90 \mathrm{E}-09$ & $3.98 \mathrm{E}-09$ & $4.08 \mathrm{E}-09$ & 4.66E-09 & 5.79E-09 & $6.78 \mathrm{E}-09$ & 7.64E-09 \\
\hline & 8.42E-09 & $1.15 \mathrm{E}-08$ & $1.57 \mathrm{E}-08$ & $1.87 \mathrm{E}-08$ & 2.12E-08 & 2.34E-08 & 3.12E-08 & 4.10E-08 & 4.77E-08 & $5.28 \mathrm{E}-08$ \\
\hline $\mathrm{Br}^{5+}$ & $1.00 \mathrm{E}-14$ & $1.00 \mathrm{E}-14$ & $1.00 \mathrm{E}-14$ & $1.00 \mathrm{E}-14$ & $1.00 \mathrm{E}-14$ & $1.00 \mathrm{E}-14$ & $1.00 \mathrm{E}-14$ & $3.81 \mathrm{E}-14$ & $1.18 \mathrm{E}-13$ & $2.84 \mathrm{E}-13$ \\
\hline & $5.68 \mathrm{E}-13$ & $4.76 \mathrm{E}-12$ & $3.30 \mathrm{E}-11$ & $9.08 \mathrm{E}-11$ & $1.76 \mathrm{E}-10$ & $2.86 \mathrm{E}-10$ & $1.08 \mathrm{E}-09$ & 3.27E-09 & $5.68 \mathrm{E}-09$ & $8.12 \mathrm{E}-09$ \\
\hline $\mathrm{Kr}^{+}$ & $1.00 \mathrm{E}-14$ & $1.00 \mathrm{E}-14$ & $1.00 \mathrm{E}-14$ & $1.00 \mathrm{E}-14$ & $1.00 \mathrm{E}-14$ & $1.01 \mathrm{E}-14$ & $1.44 \mathrm{E}-14$ & $9.96 \mathrm{E}-14$ & $4.04 \mathrm{E}-13$ & $1.02 \mathrm{E}-12$ \\
\hline & $2.00 \mathrm{E}-12$ & $1.24 \mathrm{E}-11$ & $5.35 \mathrm{E}-11$ & $1.10 \mathrm{E}-10$ & $1.74 \mathrm{E}-10$ & $2.41 \mathrm{E}-10$ & $5.81 \mathrm{E}-10$ & 1.19E-09 & $1.71 \mathrm{E}-09$ & $2.17 \mathrm{E}-09$ \\
\hline $\mathrm{Kr}^{2+}$ & $1.00 \mathrm{E}-14$ & $1.00 \mathrm{E}-14$ & $1.00 \mathrm{E}-14$ & $1.00 \mathrm{E}-14$ & $1.00 \mathrm{E}-14$ & $1.00 \mathrm{E}-14$ & $1.00 \mathrm{E}-14$ & $1.00 \mathrm{E}-14$ & $1.00 \mathrm{E}-14$ & $1.00 \mathrm{E}-14$ \\
\hline & $1.00 \mathrm{E}-14$ & $1.00 \mathrm{E}-14$ & $1.00 \mathrm{E}-14$ & $1.00 \mathrm{E}-14$ & $1.00 \mathrm{E}-14$ & $1.00 \mathrm{E}-14$ & $1.00 \mathrm{E}-14$ & $1.00 \mathrm{E}-14$ & $1.00 \mathrm{E}-14$ & $1.00 \mathrm{E}-14$ \\
\hline $\mathrm{Kr}^{3+}$ & $2.75 \mathrm{E}-09$ & 2.76E-09 & $2.77 \mathrm{E}-09$ & E-09 & 2.92E-09 & $3.00 \mathrm{E}-09$ & E-09 & E-09 & E-09 & $5.22 \mathrm{E}-09$ \\
\hline & $5.66 \mathrm{E}-09$ & 7.30E-09 & 9.34E-09 & $1.07 \mathrm{E}-08$ & $1.17 \mathrm{E}-08$ & $1.25 \mathrm{E}-08$ & $1.50 \mathrm{E}-08$ & $1.75 \mathrm{E}-08$ & $1.89 \mathrm{E}-08$ & $1.98 \mathrm{E}-08$ \\
\hline $\mathrm{Kr}^{4+}$ & $4.28 \mathrm{E}-09$ & 4.30E-09 & 4.29E-09 & 4.33E-09 & 4.41E-09 & 4.50E-09 & 5.02E-09 & 5.99E-09 & $6.80 \mathrm{E}-09$ & 7.49E-09 \\
\hline & 8.09E-09 & $1.03 \mathrm{E}-08$ & $1.32 \mathrm{E}-08$ & $1.51 \mathrm{E}-08$ & $1.67 \mathrm{E}-08$ & $1.81 \mathrm{E}-08$ & 2.32E-08 & $3.06 \mathrm{E}-08$ & $3.66 \mathrm{E}-08$ & $4.18 \mathrm{E}-08$ \\
\hline $\mathrm{Kr}^{5+}$ & $5.00 \mathrm{E}-10$ & $4.83 \mathrm{E}-10$ & 4.63E-10 & $4.58 \mathrm{E}-10$ & $4.61 \mathrm{E}-10$ & $4.69 \mathrm{E}-10$ & $5.48 \mathrm{E}-10$ & $7.72 \mathrm{E}-10$ & $1.03 \mathrm{E}-09$ & $1.29 \mathrm{E}-09$ \\
\hline & $1.55 \mathrm{E}-09$ & $2.86 \mathrm{E}-09$ & $5.22 \mathrm{E}-09$ & 7.29E-09 & $9.15 \mathrm{E}-09$ & $1.08 \mathrm{E}-08$ & $1.76 \mathrm{E}-08$ & $2.73 \mathrm{E}-08$ & $3.44 \mathrm{E}-08$ & $4.03 \mathrm{E}-08$ \\
\hline $\mathrm{Rb}^{+}$ & $0.00 \mathrm{E}+00$ & $0.00 \mathrm{E}+00$ & $0.00 \mathrm{E}+00$ & $0.00 \mathrm{E}+00$ & $0.00 \mathrm{E}+00$ & $0.00 \mathrm{E}+00$ & $0.00 \mathrm{E}+00$ & $1.42 \mathrm{E}-92$ & $4.23 \mathrm{E}-77$ & $5.61 \mathrm{E}-68$ \\
\hline & 8.43E-62 & $1.17 \mathrm{E}-46$ & $4.32 \mathrm{E}-36$ & $2.00 \mathrm{E}-31$ & $1.23 \mathrm{E}-28$ & $1.01 \mathrm{E}-26$ & $6.58 \mathrm{E}-22$ & $2.28 \mathrm{E}-18$ & $1.01 \mathrm{E}-16$ & $1.05 \mathrm{E}-15$ \\
\hline $\mathrm{Rb}^{2+}$ & $1.00 \mathrm{E}-14$ & $1.00 \mathrm{E}-14$ & $1.00 \mathrm{E}-14$ & $1.00 \mathrm{E}-14$ & $1.00 \mathrm{E}-14$ & $1.00 \mathrm{E}-14$ & $1.00 \mathrm{E}-14$ & $1.00 \mathrm{E}-14$ & $1.00 \mathrm{E}-14$ & $1.00 \mathrm{E}-14$ \\
\hline & $1.00 \mathrm{E}-14$ & $1.00 \mathrm{E}-14$ & $1.00 \mathrm{E}-14$ & $1.00 \mathrm{E}-14$ & $1.00 \mathrm{E}-14$ & $1.00 \mathrm{E}-14$ & $1.00 \mathrm{E}-14$ & $1.00 \mathrm{E}-14$ & $1.00 \mathrm{E}-14$ & $1.00 \mathrm{E}-14$ \\
\hline $\mathrm{Rb}^{3+}$ & $1.88 \mathrm{E}-09$ & $1.89 \mathrm{E}-09$ & $1.88 \mathrm{E}-09$ & $1.89 \mathrm{E}-09$ & $1.91 \mathrm{E}-09$ & $1.94 \mathrm{E}-09$ & 2.13E-09 & $2.51 \mathrm{E}-09$ & $2.85 \mathrm{E}-09$ & $3.15 \mathrm{E}-09$ \\
\hline & $3.42 \mathrm{E}-09$ & $4.46 \mathrm{E}-09$ & 5.79E-09 & $6.68 \mathrm{E}-09$ & 7.34E-09 & 7.87E-09 & $9.56 \mathrm{E}-09$ & $1.12 \mathrm{E}-08$ & $1.22 \mathrm{E}-08$ & $1.28 \mathrm{E}-08$ \\
\hline $\mathrm{Rb}^{4+}$ & 3.44E-09 & $3.46 \mathrm{E}-09$ & $3.42 \mathrm{E}-09$ & 3.39E-09 & 3.39E-09 & $3.41 \mathrm{E}-09$ & $3.56 \mathrm{E}-09$ & $3.92 \mathrm{E}-09$ & 4.23E-09 & $4.50 \mathrm{E}-09$ \\
\hline & 4.75E-09 & $5.74 \mathrm{E}-09$ & 7.22E-09 & 8.44E-09 & $9.51 \mathrm{E}-09$ & $1.05 \mathrm{E}-08$ & $1.47 \mathrm{E}-08$ & $2.16 \mathrm{E}-08$ & $2.73 \mathrm{E}-08$ & $3.24 \mathrm{E}-08$ \\
\hline $\mathrm{Rb}^{5+}$ & $1.00 \mathrm{E}-14$ & $1.00 \mathrm{E}-14$ & $1.00 \mathrm{E}-14$ & $1.00 \mathrm{E}-14$ & $1.00 \mathrm{E}-14$ & $1.00 \mathrm{E}-14$ & $1.00 \mathrm{E}-14$ & $1.60 \mathrm{E}-14$ & $4.82 \mathrm{E}-14$ & $1.13 \mathrm{E}-13$ \\
\hline & 2.23E-13 & $1.79 \mathrm{E}-12$ & $1.24 \mathrm{E}-11$ & $3.47 \mathrm{E}-11$ & $6.86 \mathrm{E}-11$ & $1.13 \mathrm{E}-10$ & 4.61E-10 & $1.52 \mathrm{E}-09$ & $2.78 \mathrm{E}-09$ & 4.12E-09 \\
\hline $\mathrm{Xe}^{+}$ & $1.52 \mathrm{E}-85$ & $3.53 \mathrm{E}-56$ & $1.56 \mathrm{E}-39$ & $3.69 \mathrm{E}-33$ & $1.23 \mathrm{E}-29$ & $2.41 \mathrm{E}-27$ & $5.06 \mathrm{E}-22$ & $1.63 \mathrm{E}-18$ & $5.39 \mathrm{E}-17$ & $4.36 \mathrm{E}-16$ \\
\hline & $1.84 \mathrm{E}-15$ & $7.25 \mathrm{E}-14$ & $1.18 \mathrm{E}-12$ & 4.44E-12 & $1.02 \mathrm{E}-11$ & $1.84 \mathrm{E}-11$ & 8.84E-11 & $3.06 \mathrm{E}-10$ & $5.59 \mathrm{E}-10$ & $8.20 \mathrm{E}-10$ \\
\hline $\mathrm{Xe}^{2+}$ & $1.00 \mathrm{E}-14$ & $1.00 \mathrm{E}-14$ & $1.00 \mathrm{E}-14$ & $1.00 \mathrm{E}-14$ & $1.00 \mathrm{E}-14$ & $1.00 \mathrm{E}-14$ & $1.00 \mathrm{E}-14$ & $1.00 \mathrm{E}-14$ & $1.00 \mathrm{E}-14$ & $1.00 \mathrm{E}-14$ \\
\hline & $1.00 \mathrm{E}-14$ & $1.00 \mathrm{E}-14$ & $1.00 \mathrm{E}-14$ & $1.00 \mathrm{E}-14$ & $1.00 \mathrm{E}-14$ & $1.00 \mathrm{E}-14$ & $1.00 \mathrm{E}-14$ & $1.00 \mathrm{E}-14$ & $1.00 \mathrm{E}-14$ & $1.00 \mathrm{E}-14$ \\
\hline $\mathrm{Xe}^{3+}$ & $6.58 \mathrm{E}-12$ & $6.35 \mathrm{E}-12$ & $5.96 \mathrm{E}-12$ & $5.74 \mathrm{E}-12$ & $5.61 \mathrm{E}-12$ & $5.53 \mathrm{E}-12$ & $5.38 \mathrm{E}-12$ & $5.40 \mathrm{E}-12$ & $5.42 \mathrm{E}-12$ & $5.43 \mathrm{E}-12$ \\
\hline & $5.42 \mathrm{E}-12$ & $5.41 \mathrm{E}-12$ & $5.39 \mathrm{E}-12$ & $5.37 \mathrm{E}-12$ & $5.37 \mathrm{E}-12$ & $5.36 \mathrm{E}-12$ & $5.35 \mathrm{E}-12$ & $5.39 \mathrm{E}-12$ & $5.41 \mathrm{E}-12$ & $5.42 \mathrm{E}-12$ \\
\hline $\mathrm{Xe}^{4+}$ & $1.25 \mathrm{E}-09$ & $1.26 \mathrm{E}-09$ & $1.26 \mathrm{E}-09$ & $1.28 \mathrm{E}-09$ & $1.30 \mathrm{E}-09$ & $1.33 \mathrm{E}-09$ & $1.50 \mathrm{E}-09$ & $1.85 \mathrm{E}-09$ & 2.17E-09 & $2.46 \mathrm{E}-09$ \\
\hline & 2.72E-09 & 3.80E-09 & $5.30 \mathrm{E}-09$ & 6.39E-09 & $7.26 \mathrm{E}-09$ & 7.99E-09 & $1.05 \mathrm{E}-08$ & 1.34E-08 & $1.51 \mathrm{E}-08$ & $1.64 \mathrm{E}-08$ \\
\hline $\mathrm{Xe}^{5+}$ & $1.00 \mathrm{E}-14$ & $1.00 \mathrm{E}-14$ & $1.00 \mathrm{E}-14$ & $1.00 \mathrm{E}-14$ & $1.00 \mathrm{E}-14$ & $1.00 \mathrm{E}-14$ & $1.00 \mathrm{E}-14$ & $2.00 \mathrm{E}-14$ & $5.90 \mathrm{E}-14$ & $1.37 \mathrm{E}-13$ \\
\hline & $2.68 \mathrm{E}-13$ & $2.17 \mathrm{E}-12$ & $1.54 \mathrm{E}-11$ & $4.39 \mathrm{E}-11$ & $8.78 \mathrm{E}-11$ & $1.46 \mathrm{E}-10$ & $6.08 \mathrm{E}-10$ & 2.03E-09 & $3.74 \mathrm{E}-09$ & $5.57 \mathrm{E}-09$ \\
\hline
\end{tabular}

Notes. Each listed rate coefficient is the largest of the radially-coupled CT rate coefficient from our MCLZ and Demkov calculations and the canonical radiative CT rate coefficient $10^{-14} \mathrm{~cm}^{3} \mathrm{~s}^{-1}$ (Butler et al. 1977; Stancil \& Zygelman 1996). Note that radiative CT does not occur for endoergic reactions. 
Table 3. Total charge transfer ionization rate coefficients $\left(\mathrm{cm}^{3} \mathrm{~s}^{-1}\right)$ for the reactions $\mathrm{X}^{0}+\mathrm{H}^{+} \rightarrow \mathrm{X}^{+}+\mathrm{H}+\delta E$.

\begin{tabular}{lcccccccccc}
\hline \hline & \multicolumn{10}{c}{$T(\mathrm{~K})$} \\
\cline { 2 - 10 } & $1.0 \mathrm{E}+02$ & $2.0 \mathrm{E}+02$ & $4.0 \mathrm{E}+02$ & $6.0 \mathrm{E}+02$ & $8.0 \mathrm{E}+02$ & $1.0 \mathrm{E}+03$ & $2.0 \mathrm{E}+03$ & $4.0 \mathrm{E}+03$ & $6.0 \mathrm{E}+03$ & $8.0 \mathrm{E}+03$ \\
Projectile & $1.0 \mathrm{E}+04$ & $2.0 \mathrm{E}+04$ & $4.0 \mathrm{E}+04$ & $6.0 \mathrm{E}+04$ & $8.0 \mathrm{E}+04$ & $1.0 \mathrm{E}+05$ & $2.0 \mathrm{E}+05$ & $4.0 \mathrm{E}+05$ & $6.0 \mathrm{E}+05$ & $8.0 \mathrm{E}+05$ \\
\hline $\mathrm{Ge}^{0}$ & $1.01 \mathrm{E}-12$ & $1.01 \mathrm{E}-12$ & $1.01 \mathrm{E}-12$ & $1.01 \mathrm{E}-12$ & $1.01 \mathrm{E}-12$ & $1.01 \mathrm{E}-12$ & $1.01 \mathrm{E}-12$ & $1.01 \mathrm{E}-12$ & $1.01 \mathrm{E}-12$ & $1.01 \mathrm{E}-12$ \\
& $1.01 \mathrm{E}-12$ & $1.01 \mathrm{E}-12$ & $1.01 \mathrm{E}-12$ & $1.01 \mathrm{E}-12$ & $1.01 \mathrm{E}-12$ & $1.01 \mathrm{E}-12$ & $1.01 \mathrm{E}-12$ & $1.18 \mathrm{E}-12$ & $2.32 \mathrm{E}-12$ & $5.83 \mathrm{E}-12$ \\
$\mathrm{Se}^{0}$ & $1.01 \mathrm{E}-12$ & $1.01 \mathrm{E}-12$ & $1.01 \mathrm{E}-12$ & $1.01 \mathrm{E}-12$ & $1.01 \mathrm{E}-12$ & $1.01 \mathrm{E}-12$ & $1.01 \mathrm{E}-12$ & $1.01 \mathrm{E}-12$ & $1.01 \mathrm{E}-12$ & $1.02 \mathrm{E}-12$ \\
& $1.04 \mathrm{E}-12$ & $1.86 \mathrm{E}-12$ & $1.24 \mathrm{E}-11$ & $4.24 \mathrm{E}-11$ & $9.51 \mathrm{E}-11$ & $1.71 \mathrm{E}-10$ & $8.41 \mathrm{E}-10$ & $3.11 \mathrm{E}-09$ & $6.00 \mathrm{E}-09$ & $9.19 \mathrm{E}-09$ \\
$\mathrm{Br}^{0}$ & $1.01 \mathrm{E}-12$ & $1.01 \mathrm{E}-12$ & $1.01 \mathrm{E}-12$ & $1.02 \mathrm{E}-12$ & $1.04 \mathrm{E}-12$ & $1.09 \mathrm{E}-12$ & $2.37 \mathrm{E}-12$ & $1.48 \mathrm{E}-11$ & $4.42 \mathrm{E}-11$ & $9.07 \mathrm{E}-11$ \\
& $1.52 \mathrm{E}-10$ & $6.28 \mathrm{E}-10$ & $1.98 \mathrm{E}-09$ & $3.51 \mathrm{E}-09$ & $5.05 \mathrm{E}-09$ & $6.58 \mathrm{E}-09$ & $1.36 \mathrm{E}-08$ & $2.50 \mathrm{E}-08$ & $3.45 \mathrm{E}-08$ & $4.29 \mathrm{E}-08$ \\
$\mathrm{Kr}^{0}$ & $3.62 \mathrm{E}-45$ & $1.80 \mathrm{E}-31$ & $1.42 \mathrm{E}-23$ & $1.62 \mathrm{E}-20$ & $8.27 \mathrm{E}-19$ & $1.09 \mathrm{E}-17$ & $4.93 \mathrm{E}-15$ & $3.27 \mathrm{E}-13$ & $2.14 \mathrm{E}-12$ & $6.70 \mathrm{E}-12$ \\
& $1.48 \mathrm{E}-11$ & $1.17 \mathrm{E}-10$ & $5.70 \mathrm{E}-10$ & $1.22 \mathrm{E}-09$ & $1.96 \mathrm{E}-09$ & $2.75 \mathrm{E}-09$ & $6.81 \mathrm{E}-09$ & $1.41 \mathrm{E}-08$ & $2.04 \mathrm{E}-08$ & $2.59 \mathrm{E}-08$ \\
$\mathrm{Rb}^{0}$ & $1.00 \mathrm{E}-14$ & $1.00 \mathrm{E}-14$ & $1.00 \mathrm{E}-14$ & $1.00 \mathrm{E}-14$ & $1.00 \mathrm{E}-14$ & $1.00 \mathrm{E}-14$ & $1.00 \mathrm{E}-14$ & $1.00 \mathrm{E}-14$ & $1.00 \mathrm{E}-14$ & $1.00 \mathrm{E}-14$ \\
& $1.00 \mathrm{E}-14$ & $1.00 \mathrm{E}-14$ & $1.00 \mathrm{E}-14$ & $1.00 \mathrm{E}-14$ & $1.00 \mathrm{E}-14$ & $1.00 \mathrm{E}-14$ & $1.00 \mathrm{E}-14$ & $1.00 \mathrm{E}-14$ & $1.01 \mathrm{E}-14$ & $1.12 \mathrm{E}-14$ \\
$\mathrm{Xe}^{0}$ & $1.00 \mathrm{E}-14$ & $1.00 \mathrm{E}-14$ & $1.00 \mathrm{E}-14$ & $1.00 \mathrm{E}-14$ & $1.00 \mathrm{E}-14$ & $1.00 \mathrm{E}-14$ & $1.00 \mathrm{E}-14$ & $1.04 \mathrm{E}-14$ & $1.48 \mathrm{E}-14$ & $3.34 \mathrm{E}-14$ \\
& $8.32 \mathrm{E}-14$ & $1.60 \mathrm{E}-12$ & $1.91 \mathrm{E}-11$ & $6.51 \mathrm{E}-11$ & $1.42 \mathrm{E}-10$ & $2.49 \mathrm{E}-10$ & $1.13 \mathrm{E}-09$ & $3.78 \mathrm{E}-09$ & $6.84 \mathrm{E}-09$ & $9.99 \mathrm{E}-09$ \\
\hline
\end{tabular}

Notes. For Ge, Se, and Br, the spin-orbit coupling rate coefficient found for $\mathrm{Cl}^{+}, 10^{-12} \mathrm{~cm}^{3} \mathrm{~s}^{-1}$ (Pradhan \& Dalgarno 1994), is adopted as an upper limit when the rate from radial coupling falls below that value. SO coupling is not relevant for $\mathrm{CT}$ reactions involving neutral $\mathrm{Kr}$, $\mathrm{Rb}$, and $\mathrm{Xe}$, and hence we set the rate coefficient to the canonical radiative CT rate $\left(10^{-14} \mathrm{~cm}^{3} \mathrm{~s}^{-1}\right.$; Butler et al. 1977) when the radial coupling rate coefficient is smaller than that value. Note that radiative $\mathrm{CT}$ does not occur for the endoergic reaction $\mathrm{Kr}^{0}+\mathrm{H}^{+} \rightarrow \mathrm{Kr}^{+}+\mathrm{H}+\delta E$.

of these systems. Our forthcoming study of the sensitivity of $n$-capture abundance determinations to atomic data uncertainties, using Monte Carlo simulations with photoionization modeling codes, will help to identify the ions that most urgently require QMOCC determinations of CT rate coefficients.

\section{Summary}

We have presented CT recombination rate coefficients for the first five ionization stages of the $n$-capture elements $\mathrm{Ge}, \mathrm{Se}$, $\mathrm{Br}, \mathrm{Kr}, \mathrm{Rb}$, and Xe reacting with neutral hydrogen. A multichannel Landau-Zener approach was employed for multiplycharged ions, while the Demkov approximation was used for singly-charged species. We note that the computed rate coefficients for $q=5$ are lower limits, due to the incompleteness of energy listings in the NIST database. Because of that, we recommend that the four-times ionized recombination rate coefficients be used for $q \geq 5$, with the exception of $\mathrm{Ge}^{5+}$. For reactions between the neutral species of these elements and $\mathrm{H}^{+}$, we computed CT ionization rate coefficients. Total rate coefficients are given over the range of temperatures $10^{2}-10^{6} \mathrm{~K}$ in Tables 2 and 3 , while final-state resolved rate coefficients are presented in Tables 4 and 5 (available at the CDS). In addition, all rate coefficients can be obtained at http://www . pa.msu.edu/astro/ atomicdata/charge_transfer/. In tandem with our concurrent investigations into the photoionization and radiative and dielectronic recombination properties of $n$-capture elements (e.g., Sterling \& Witthoeft 2011; Sterling 2011), these CT rate coefficient determinations will enable the abundances of $n$-capture elements to be reliably determined in ionized astrophysical nebulae. Such studies bear significant implications for $n$-capture nucleosynthesis, the structure and internal mixing of evolved stars, and the chemical evolution of trans-iron elements in the Universe.

Acknowledgements. N. C. Sterling gratefully acknowledges support from an NSF Astronomy and Astrophysics Postdoctoral Fellowship under award AST0901432 and from NASA grant 06-APRA206-0049. The work of P. C. Stancil was partially supported by NASA grant NNX09AC46G.

\section{References}

Baldwin, J. A., Verner, E. M., Verner, D. A., et al. 2000, ApJS, 129, 229 Blum, R. D., \& McGregor, P. J. 2008, AJ, 135, 1708

Busso, M., Gallino, R., \& Wasserburg, G. J. 1999, ARA\&A, 37, 239

Butler, S. E., \& Dalgarno, A. 1979, ApJ, 234, 765

Butler, S. E., \& Dalgarno, A. 1980a, A\&A, 85, 144

Butler, S. E., \& Dalgarno, A. 1980b, ApJ, 241, 838

Butler, S. E., Guberman, S. L., \& Dalgarno, A. 1977, Phys. Rev. A, 16, 500

Chang, A. M., \& Pritchard, D. E. 1979, J. Chem. Phys., 70, 4524

Dalgarno, A., Du, M. L., \& You, J. H. 1990, ApJ, 349, 675

Demkov, Y. N. 1964, Sov. Phys.-JETP, 18, 138

Dinerstein, H. L. 2001, ApJ, 550, L223

Froese Fischer, C. 1977, The Hartree-Fock Method for Atoms: A Numerical Approach (New York: Wiley)

Gentry, W. R., \& Giese, C. F. 1977, J. Chem. Phys., 67, 2355

Herzberg, G. 1952, Spectra of Diatomic Molecules (New York: Van Nostrand), 318

Janev, R. K., Belic, D. S., \& Bransden, B. J. 1983, Phys. Rev. A, 28, 1293

Kimura, M., \& Lane, N. F. 1989, Ad. At. Mol. Opt. Phys., 26, 79

Kingdon, J. B. 1995, MNRAS, 274, 425

Kingdon, J. B., \& Ferland, G. J. 1996, ApJS, 106, 205

Lide, D. R. 2002, CRC Handbook of chemistry and physics: a ready-reference book of chemical and physical data (Boca Raton: CRC Press)

Olson, R. E. 1972, Phys. Rev. A, 6, 1822

Otsuka, M., Tajitsu, A., Hyung, S., \& Izumiura, H. 2010, ApJ, 723, 658

Otsuka, M., Meixner, M., Riebel, D., et al. 2011, ApJ, 729, 39

Péquignot, D., \& Baluteau, J. P. 1994, A\&A, 283, 593

Pradhan, A., \& Dalgarno, A. 1994, Phys. Rev. A, 49, 960

Sharpee, B., Zhang, Y., Williams, R., et al. 2007, ApJ, 659, 1265

Sneden, C., Cowan, J. J., \& Gallino, R. 2008, ARA\&A, 46, 241

Stancil, P. C. 2001, in Spectroscopic Challenges of Photoionized Plasmas, ed. G. J. Ferland, \& D. W. Savin, ASP Conf. Ser., 247, 3

Stancil, P. C., \& Zygelman, B. 1996, ApJ, 472, 102

Sterling, N. C. 2011, A\&A, 533, A62

Sterling, N. C., \& Dinerstein, H. L. 2003, Rev. Mex. Astron. Ser. Conf., 18, 133

Sterling, N. C., \& Dinerstein, H. L. 2008, ApJS, 174, 158

Sterling, N. C., \& Witthoeft, M. C. 2011, A\&A, 529, A147

Sterling, N. C., Dinerstein, H. L., \& Bowers, C. W. 2002, ApJ, 578, L55

Sterling, N. C., Dinerstein, H. L., \& Kallman, T. R. 2007, ApJS, 169, 37

Sterling, N. C., Dinerstein, H. L., Hwang, S., et al. 2009, PASA, 26, 339

Swartz, D. A. 1994, ApJ, 428, 267

Vanzi, L., Cresci, G., Telles, E., \& Melnick, J. 2008, A\&A, 486, 393

Wang, J. G., Stancil, P. C., Turner, A. R., \& Cooper, D. L. 2004, Phys. Rev. A, 69, 062702

Zhang, Y., Liu, X.-W., Luo, S.-G., Péquignot, D., \& Barlow, M. J. 2005, A\&A, 442, 249

Zygelman, B., Cooper, D. L., Ford, M. J., et al. 1992, Phys. Rev. A, 46, 3846 